\title{
Dynamique de l'eau dans le sol en forêt tropicale humide guyanaise. Influence de la couverture pédologique
}

\author{
J.M. GUEHL \\ I.N.R.A., Station de Sylviculture et de Production \\ Centre de Recherches forestieres de Nancy \\ Champenoux, F 54280 Seichamps
}

\begin{abstract}
Résumé
En Guyane française septentrionale l'étude détaillée de la couverture pédologique a permis de mettre en évidence l'existence de systèmes à forte différenciation latérale qui ont été interprétés comme des systèmes de transformation d'une couverture ferrallitique initiale suivant un déterminisme d'origine tectonique mais d’amplitude pédologique (BoulET, et $(a l$, , 1979).

Cet article présente les premiers résultats d'une étude des répercussions de cette variabilité du milieu sur les termes du bilan hydrique dans le sol. Il est basé sur une étude du fonctionnement hydrique in situ (mesures neutroniques et tensiométriques) dans un système pédologique mixte en situation de pente légère (sols sur pegmatite).

En saison des pluies, on note dans la couverture initiale ( $\mathrm{I}_{\bar{i}}$, fig. 3 ), en relation avec la macroporosité importante (fig. 7) des horizons à microagrégats 1 et 2 (fig. 3) et un coefficient de perméabilité (méthode de Muntz) restant supérieur à $3 \mathrm{~mm} \mathrm{~h}^{-1}$ dans les deux premiers mètres de sol (fig. 5), un drainage vertical en conditions non saturées même durant les périodes de très fortes précipitations (fig. 8, 10).

De $I_{4}$ à $I_{0}$, le rapprochement vers la surface de l'horizon 6 (incision de la surlace topographique initiale), de densité apparente élevée (tabl. 1), présentant une faible macroporosité (fig. 7) et des valeurs du coefficient de Muntz voisines de 0 (fig. 5), provoque l'apparition d'une nappe perchée (fig. 8) caractérisée par un écoulement latéral efficace (fig. 10 et 12) grâce à la configuration interne favorable du sommet de l'horizon 6 .

Au cours des 2 saisons sèches étudiées (petit été de mars 1981 et grande saison sc̀che d'août-novembre 1980), caractérisées par d'importants déficits hydriques climatiques (tabl. 3), le réservoir sol est très largement utilisé pour la satisfaction des besoins hydriques de la forêt (tabl. 4 et fig. 13). Deux types bien tranchés d'évolution des profils hydriques en profondeur apparaissent :

- Dans le cas des sites amont ( $I_{5}$ à $I_{2}$, fig. 3), les variations d'humidité encore très importantes à $165 \mathrm{~cm}$ de profondeur attestent d’une extraction racinaire dépassant la profondeur des tubes d'accès.

- Dans le cas du site $I_{1}$ les variations profondes d'humidité sont nettement plus faibles en raison de la présence en profondeur de l'horizon 6 réduisant les possibilités de développement du système racinaire.

Dans de telles conditions d'extraction racinaire profonde, il n'a pas été possible d'estimer l'évapotranspiration réelle de la forêt à partir des mesures neutroniques, mais les modalités expérimentales devant permettre une telle estimation pour le milieu étudié sont précisćes.
\end{abstract}




\section{Introduction}

En raison de la forte pluviométric caractérisant le climat de la Guyane (de type subéquatorial avec rarement moins de $2500 \mathrm{~mm}$ annuels d'eau), on peut être tenté de penser que le facteur hydrique ne constitue pas un facteur limitant de la production végétale. Ce serait une représentation simpliste et fausse de la réalité.

En effet si le bilan hydrique climatique annuel est toujours excédentaire, la caractéristique principale du climat guyanais est celle d'une alternance de périodes d'importants excès d'eau avec risques d'engorgement des sols et de périodes de déficit hydrique (petite saison sèche en févricl ou mars et grande saison sèche d'août-novembre) pouvant exercer un effet dépressif sensible sur les végétaux (Fougkrouze, 1966). Ainsi, dans la zone de la présente étude expérimentale, Prevost \& Puig (1981) ont noté une diminution importante de laceroissement diamétral des arbres de la forêt primaire et d'une forêt secondáre âgée de 6 ans lors de la grande saison sèche de 1979.

Les variations des caractéristiques hydriques des sols constituent un important facteur de modulation de l'action du facteur hydrique sur les végétaux (AuSSENAC \& Granier, 1979; Ballit, 1980).

Le but de l'étude dont nous présentons les premiers résultats ici était de préciser l'influence de telles variations sur le fonctionnement hydrique in situ du sol dans l'un des systèmes pédologiques à forte différenciation latérale mis en évidence dans la région des schistes Bonidoro (Humbis. 1978 ; Boulet, 1981). L'existence de tels systèmes a également été établic dans d'autres zones de la Guyane septentrionale (BOulet, 1978 ; Humbel, 1978 ; Boullet et al.. 1979).

Ce travail a été réalisé dans le cadre de l'opération ECEREX (ECologie, ERosion, EXpérimentation) démarrée en 1976, qui a pour objet l'étude de l'écosystème forestier guyanais et de son comportement lace à différents modes de mise en valeur. Cette opération est axée sur l'étude de 10 bassins versants expérimentaux (SARralth, 1980).

\section{Méthodologie d'étude et site d'expérimentation}

\subsection{Rappel théorique sur la dynanique de l'eau dans le sol}

Dans le système poreux sol, la force motrice des flux d'eau est constituéc par le gradient de l'énergie potentielle de l'eau. Le potentiel de l'eau dans le sol exprime l'énergic de liaison de l'eau dans un système relativement à celle de l'eau libre dans les mêmes conditions de température et d'altitude (HILlEL, 1974). Nous avons rapporté cette différence d'énergie potenticlle à l'unité de volume d'eau, cela confère au potentiel les dimensions d'une pression (millibars), numériquement les grandeurs ainsi exprimées sont sensiblement égales à une hauteur d'eau exprimée en cm.

Le potentiel total $\left(\mathrm{T}_{\mathrm{t}}\right)$ de l'eau fait intervenir de façon additive un terme gravitationnel $\Psi_{m}$ lié aux variations d'altitude et compté négativement vers le bas et un 
terme d'état pouvant être soit négatif (potentiel matriciel dû aux forces de rétention capillaire et d'adsorption à la surface des particules), soit positif et exprimant alors une pression hydrostatique. Pour des raisons de commodité, ces deux termes d'état de nature physique différente seront désignés indistinctement par $\Psi_{m}$, ce terme pouvant prendre des valeurs négatives (domaine de l'eau liée) ou positives (domaine de pression hydrostatique) sans solution de continuité pour $\Psi_{m}=0$ (eau libre).

$$
\text { On a donc : } \Psi_{t}=\Psi_{g}+\Psi_{m}
$$

Le potenticl hydrique a été mesuré à l'aide de tensiomètres (appareils de type classique soll moIsTure à manomètre à mercure). Le principe de cette mesure (fig. 1) repose sur l'établissement d'un équilibre hydrostatique entre l'eau du sol, l'eau à l'intérieur de la bougie poreuse et le manomètre. Cet équilibre est décrit par les équations suivantes (on adopte les symboles de la fig. 1) :

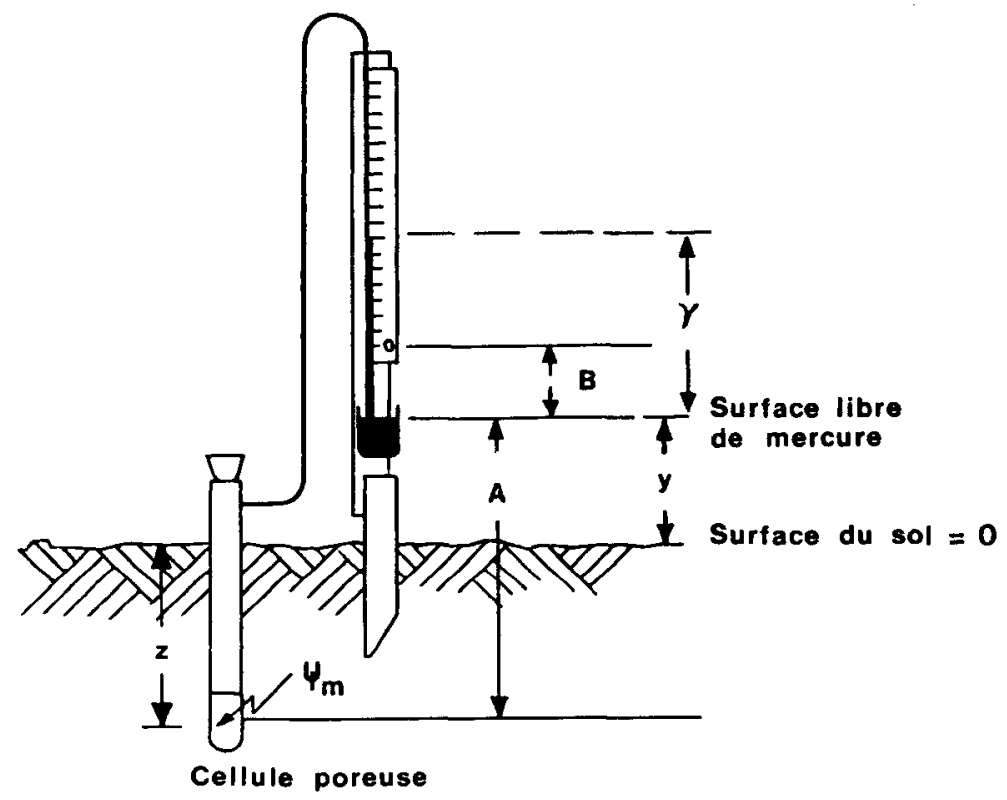

Fig. 1

Schéma de principe du fonctionnement d'un tensiomètre.

Principle of functionning of a tensiometer.

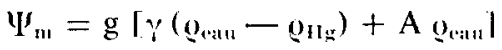

$$
\begin{aligned}
& \Psi_{k}=-0_{* a 11} \mathrm{~g} \mathrm{z}
\end{aligned}
$$

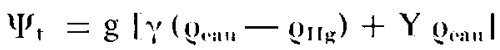

où $\underline{Q}_{\text {ryg }}$ et $\underline{0}_{\text {wan }}$ sont respectivement les masses volumiques du mercure et de l'eau, $g$ l'accélération de la pesanteur. 
Lorsque $\Psi_{\jmath n}$ devient inféricur à $-800 \mathrm{mb}$, il y a accroissement rapide de l'entrée d'air et désamorçage du système dont l'utilisation reste donc confinée à des situations de polentiel hydrique relativement élevé.

Le long d'un axe $O x$, la densité du flux hydrique instantané traversant un élément de surface normal à Ox est reliée au gradient de $\Psi_{t}$ par la loi de Darcy étendue aux conditions non saturées :

$$
\Phi_{\mathrm{x}}=-\mathrm{K}_{\mathrm{x}}(\theta) \frac{\partial \Psi_{\mathrm{t}}}{\partial \mathrm{x}}
$$

où $K_{x}$ est la conductivité hydraulique du sol dans la direction Ox et " l'humidité volumique du sol $\left(\mathrm{cm}^{3} / \mathrm{cm}^{3}\right)$. Le signe — indique une circulation dans le sens des potentiels décroissants.

Suivant la direction verlicale, cette relation devient par combinaison avec (1) : $\boldsymbol{\Phi}_{z}=-\mathbf{K}_{\%}(\theta) \frac{\partial \Psi_{t}}{\partial z}=-K_{z}(1)\left(\frac{\partial \Psi_{m}^{\prime}}{\partial z}+\frac{\partial \Psi_{z}}{\partial z}\right)=-K_{\%}(\theta) \frac{\partial \Psi_{m}}{\partial z}-K_{z}(0) g_{c a u} g$

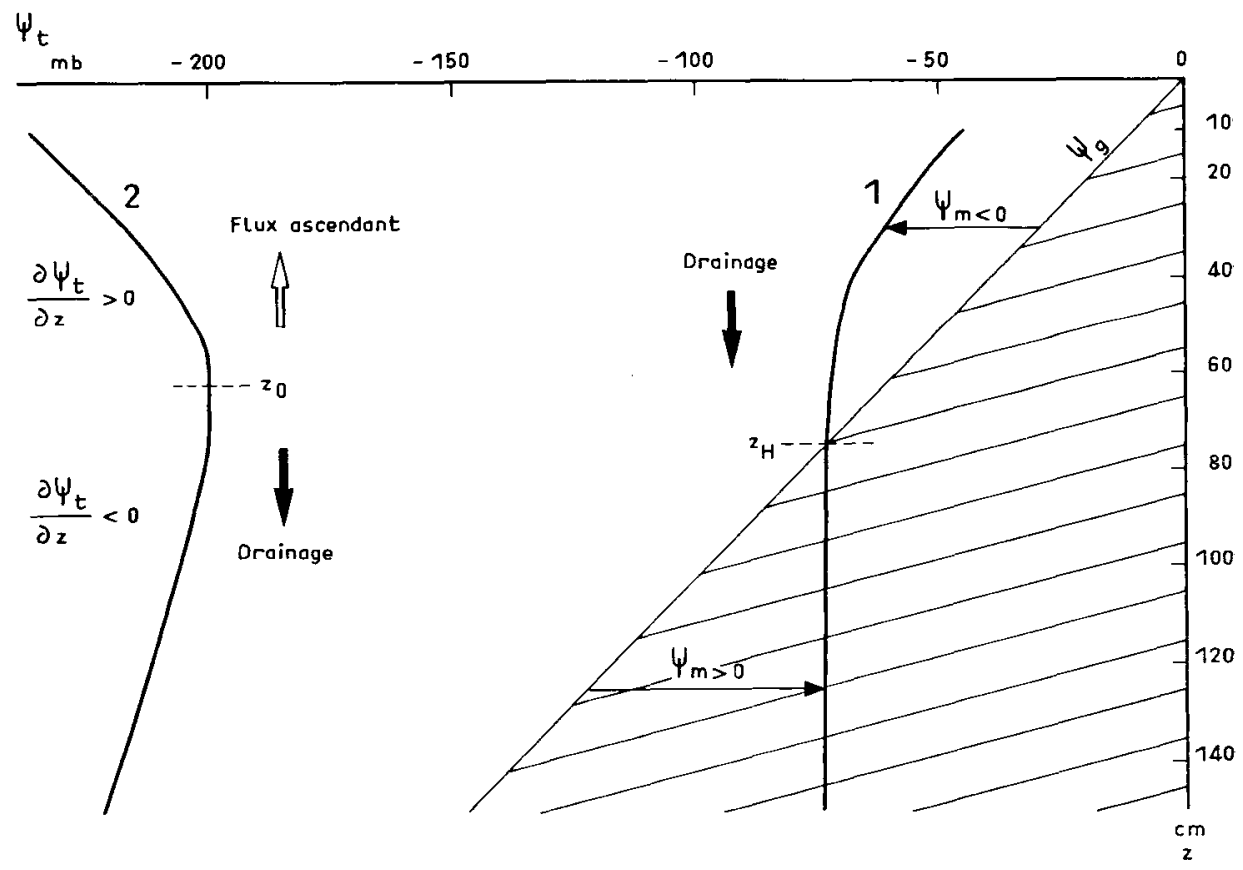

FIG. 2

Profils verticaux du potentiel hydrique total $\left(\Psi_{\prime}\right)$ et interprétation hydrodynamique. Vertical profiles of soil total water potential $\left(\Psi_{i}\right)$ and hydrodynamic signification.

$\mathbf{z}_{0} \quad$ : niveau du plan de flux nul.

$\mathrm{z}_{\|} \quad$ : niveau de la nappe.

$\Psi_{11}<0:$ potentiel matricicl.

$\Psi_{11}>{ }_{11}:$ potentiel hydrostatique.

$\Psi_{t}^{\prime}=\Psi_{: \underline{m}}+\Psi_{\mathrm{III}}$. 
La figure 2 montre 2 exemples caractéristiques de profils verticaux de $\Psi_{1}$. Les équations (6) en permettent une interprétation hydrodynamique :

- Le profil 1, obtenu en période de fortes précipitations, se caractérise par l'existence à la profondeur $z_{I I}$ d'un niveau de potentiel matriciel $\Psi_{m}$ nul sous lequel où $K_{x}$ est la conductivité hydraulique du sol dans la direction $O x$ et $\theta$ l'humidité exactement compensées par les variations de pression hydrostatique), on se trouve en présence d'une nappe d'eau sans écoulement vertical perceptible. Au-dessus du niveau $z_{11}$, on est dans le domaine de l'eau liée $\left(\Psi_{m}<0\right)$, l'existence d'un gradient négatif vers le bas indique une alimentation de la nappe par drainage en milieu non saturé.

- Le profil 2 est totalement hors saturation. A la profondeur $z_{0,}$, un plan de flux nul $\left(\frac{\partial \Psi_{1}}{\partial z}=0\right)$ partage le sol en 2 zones hydrodynamiquement indépendantes. Au-dessus de $\mathrm{z}_{0}$, existe un flux ascendant lié all flux évapotranspiratoire, en dessous de $z_{w}$, il y a drainage.

En milieu isotrope, l'équation (5) se généralise aux 3 dimensions à l'aide de lopérateur gradient $(\mathbb{V})$ :

$$
\text { W }=-K(0) \nabla\left(\Psi_{1}\right)
$$

Les flux sont alors normaux aux lignes équipotentielles et dirigés vers les potentiels décroissants.

L'équation (7) combinée à l'équation de conservation de la masse :

$$
\frac{\partial \theta}{\partial t}=-V(\phi)
$$

donne localement l'équation différentielle générale de l'écoulement :

$$
\frac{\partial \theta}{\partial t}=\nabla\left\{K(\theta) \vee\left(\Psi_{1}\right) !\right.
$$

où $\mathrm{t}$ représente le temps.

En milieu saturé incompressible $\frac{\partial 0}{\partial t}=0$ el $\mathrm{K}$ est constant et égal à une valeur maximale $K_{s}$ pour laquelle l'ensemble de l'espace poral participe à l'écoulement.

\subsection{Description du site expérimental}

\subsection{L'environnement pédologique}

Une analyse structurale détaillée de l'organisation des couvertures pédologiques des dix bassins versants ECEREX a permis de préciser les relations spatiales entre des couvertures pédologiques à drainage vertical libre (DVL) et des couvertures dites à drainage bloqué (DVB) (Boulet et al., 1979) dans le domaine des schistes Bonidoro et de proposer une interprétation génétique de leur différenciation (BouLET, 1981). 


\section{TABleaU 1}

Densité apparente sèche (da) et humidité pondérale ( $H_{p}$ en $\%$ ) ainsi qu'humidité volumique $(\theta v=d a . H p$ en $\%)$

au pF 4,2 poür les différents tubes d'accès netutronique.

\begin{tabular}{|c|c|c|c|c|c|c|c|c|c|}
\hline \multirow{2}{*}{$\begin{array}{l}\text { Profon- } \\
\text { deur } \\
\mathrm{cm}\end{array}$} & \multicolumn{3}{|c|}{$\mathrm{NI}_{11}$} & \multicolumn{3}{|c|}{$\mathrm{NI}_{12}$} & \multicolumn{3}{|c|}{$\mathrm{NI}_{21}$} \\
\hline & $\mathrm{Hp}$ & da & $\theta v$ & $\mathrm{Hp}$ & $\mathrm{da}$ & $\theta \mathrm{v}$ & $\mathrm{Hp}$ & $\mathrm{da}$ & $\theta \mathrm{v}$ \\
\hline 15 & 7,99 & 1,15 & 9.19 & 8,15 & 1.15 & 9,37 & 8,94 & 1,15 & 10,28 \\
\hline 25 & 7,83 & 1,20 & 9,40 & 8.93 & 1,20 & 10,72 & 9,07 & 1,20 & 10,88 \\
\hline 35 & 9,88 & 1,35 & 13,34 & 7,39 & 1,35 & 9,98 & 9,96 & 1,30 & 12,55 \\
\hline 45 & 11,54 & 1,40 & 16,16 & 8,91 & 1,35 & 12,03 & 11,30 & 1,35 & 15,26 \\
\hline 55 & 13,71 & 1,45 & 19,88 & 10,67 & 1,35 & 14,40 & 12,29 & 1,35 & 16,59 \\
\hline 65 & 9,80 & 1.45 & 14,21 & 12,42 & 1,40 & 17,39 & 13,11 & 1,35 & 17,70 \\
\hline 75 & 8,19 & 1,50 & 12,25 & 11.49 & 1.45 & 16,66 & 11,94 & 1.40 & 16,72 \\
\hline 85 & 5,71 & 1,55 & 8,85 & 9,30 & 1.45 & 13,49 & 12,000 & 1,40 & 16,80 \\
\hline 95 & 8,69 & 1,60 & 13,90 & 8.07 & 1,50 & 12,11 & 11,11 & 1,40 & 15,55 \\
\hline 105 & 8,80 & 1.60 & 14,08 & 8,27 & 1,55 & 12,82 & 9.67 & 1.50 & 14,51 \\
\hline 115 & 9,32 & 1.60 & 14.91 & 8.03 & 1,60 & 12,85 & 8,08 & 1,60 & 12.93 \\
\hline 125 & 10,23 & 1,60 & 16,37 & 4,83 & 1,60 & 7,13 & 7,44 & 1,60 & 11,90 \\
\hline 135 & 9.44 & 1.60 & 13.50 & 7,53 & 1.60 & 12,05 & 7.24 & 1,60 & 11,58 \\
\hline 145 & 7,39 & 1.60 & 11,82 & 7,35 & 1,60 & 11.76 & 7,41 & 1.60 & 11,86 \\
\hline 155 & 7,49 & 1,60 & 11.98 & 7,07 & 1,60 & 11,31 & 7,45 & 1,60 & 11,92 \\
\hline 165 & 7,72 & 1.60 & 12.35 & 7,82 & 1.60 & 12.51 & 10,55 & 1.60 & 16,88 \\
\hline
\end{tabular}

Les valeurs en italique correspondent à la profondeur d'apparition du matériau d'altération de la pegmatite.

The values in italics correspond to the depth of the altered pegmatite rock material. 
Bulk density (da) and soil water content ( $\mathrm{Hp} \mathrm{g} / \mathrm{g}$ and $\left.\theta \mathrm{vg} / \mathrm{cm}^{3}\right)$ at $p F 4.2$ for the different neutron tubes.

\begin{tabular}{|c|c|c|c|c|c|c|c|c|}
\hline \multicolumn{3}{|c|}{$\mathrm{NI}_{2,2}$} & \multicolumn{3}{|c|}{$\mathrm{NI}_{31}$} & \multicolumn{3}{|c|}{$\mathrm{NI}_{32}$} \\
\hline $\mathrm{Hp}$ & $\mathrm{da}$ & $\theta \mathrm{v}$ & $\mathrm{Hp}$ & da & $\theta \mathrm{v}$ & Hp & $\mathrm{da}$ & $\theta \mathrm{v}$ \\
\hline 8,55 & 1,15 & 9,83 & 9,70 & 1,15 & 11,16 & 8,66 & 1,15 & 9,96 \\
\hline 9,97 & 1,20 & 11,96 & 8,61 & 1,20 & 10,33 & 8,70 & 1,20 & 10,44 \\
\hline 11,12 & 1,30 & 14,46 & 9,80 & 1,20 & 11,76 & 11,43 & 1.20 & 13,72 \\
\hline 12.52 & 1.35 & 16,90 & 10,81 & 1,30 & 14,05 & 12,18 & 1,30 & 15,83 \\
\hline 12,44 & 1,35 & 16,75 & 11,76 & 1,35 & 15,88 & 12,55 & 1,35 & 16,94 \\
\hline 12,34 & 1,35 & 16,66 & 13,84 & 1,35 & 18,68 & 12,99 & 1,35 & 17,54 \\
\hline 12,12 & 1.40 & 16,97 & 13,98 & 1,35 & 18,87 & 13,21 & 1,35 & 17,83 \\
\hline 14,31 & 1,40 & 20,03 & 13,82 & 1,35 & 18,66 & 13,84 & 1,35 & 18,68 \\
\hline 12,39 & 1,40 & 17,35 & 13,27 & 1,35 & 17,91 & 13,83 & 1,40 & 19,36 \\
\hline 11,34 & 1,50 & 17,01 & 14,13 & 1,35 & 19,08 & 10,04 & 1,40 & 14,06 \\
\hline 9,61 & 1,60 & 15,38 & 9,11 & 1,35 & 12,30 & 10,99 & 1,45 & 15,94 \\
\hline 8,04 & 1,60 & 12,86 & 11,25 & 1,50 & 16,88 & 9,26 & 1,50 & 13,89 \\
\hline 7,31 & 1,60 & 11,70 & 9,28 & 1,50 & 13,92 & 7,90 & 1,55 & 12,25 \\
\hline 6,43 & 1,60 & 10,29 & 7,55 & 1,60 & 12,08 & 6,75 & 1,60 & 10,80 \\
\hline 6,60 & 1,60 & 10,56 & 7,44 & 1,65 & 12,28 & 6,23 & 1,60 & 9,97 \\
\hline 7,51 & 1,60 & 12,02 & 6,79 & 1,65 & 11,20 & 6,06 & 1,65 & 10,00 \\
\hline
\end{tabular}


Tableau 1 (suite)

\begin{tabular}{|c|c|c|c|c|c|c|c|c|c|}
\hline \multirow{2}{*}{$\begin{array}{l}\text { Profon- } \\
\text { deur } \\
\mathrm{cm}\end{array}$} & \multicolumn{3}{|c|}{$\mathrm{NI}_{+1}$} & \multicolumn{3}{|c|}{$\mathrm{NI}_{42}$} & \multicolumn{3}{|c|}{$\mathrm{NI}_{51}$} \\
\hline & $\mathrm{Hp}$ & $\mathrm{da}$ & $\theta \mathrm{v}$ & $\mathrm{Hp}$ & $\mathrm{da}$ & $0 \mathrm{v}$ & $\mathrm{Hp}$ & $\mathrm{da}$ & $\theta \mathrm{v}$ \\
\hline 15 & 9,63 & 1,15 & 11,07 & 9.40 & 1.15 & 10,81 & 10,22 & 1,15 & 11.75 \\
\hline 25 & 9,52 & 1,20 & 11,42 & 9,97 & 1.20 & 11,96 & 9,58 & 1,20 & 11,98 \\
\hline 35 & 9,59 & 1,20 & 11,51 & 11,79 & 1,20 & 14,15 & 10,82 & 1,80 & 12,98 \\
\hline 45 & 10,54 & 1,30 & 13,70 & 10,82 & 1.30 & 14,07 & 12,30 & 1.30 & 15,99 \\
\hline 55 & 11,16 & 1,30 & 14,51 & 12,41 & 1.30 & 16,13 & 11,73 & 1,35 & 15.84 \\
\hline 65 & 11,56 & 1,35 & 15,61 & 12,55 & 1,35 & 16,94 & 11,79 & 1,35 & 15,92 \\
\hline 75 & 13,15 & 1,35 & 17,75 & 12.99 & 1.35 & 17,54 & 12,04 & 1,35 & 16,25 \\
\hline 85 & 13,59 & 1,35 & 18,35 & 13,29 & 1,35 & 17,94 & 12,99 & 1,35 & 17,54 \\
\hline 95 & 13,62 & 1,35 & 18,39 & 13,81 & 1,35 & 18,64 & 12,87 & 1,35 & 17,37 \\
\hline 105 & 13,84 & 1,35 & 18,68 & 13,67 & 1,35 & 18,45 & 13,73 & 1,35 & 18,54 \\
\hline 115 & 13,98 & 1,35 & 18,87 & 13,82 & 1,35 & 18,66 & 13,71 & 1,35 & 18,51 \\
\hline 125 & 14,46 & 1,35 & 19,52 & 14,55 & 1,40 & 20,37 & 14,12 & 1,35 & 19,06 \\
\hline 135 & 14,57 & 1,40 & 20,40 & 11,43 & 1,40 & 16,00 & 13,84 & 1,35 & 18,68 \\
\hline 145 & 10,76 & 1,50 & 16,14 & 9,21 & 1,50 & 13,82 & 13,70 & 1,35 & 18,50 \\
\hline 155 & 11,31 & 1,60 & 18,10 & 8,82 & 1,60 & 14,11 & 13,32 & 1,35 & 17,98 \\
\hline 165 & 9,75 & 1,65 & 16,09 & 8,41 & 1,65 & 13,88 & 12,43 & 1,35 & 16,78 \\
\hline
\end{tabular}




\begin{tabular}{|c|c|c|c|c|c|c|c|c|c|c|c|}
\hline \multicolumn{3}{|c|}{$N I_{i=2}$} & \multicolumn{3}{|c|}{$N I_{\tilde{i}: 3}$} & \multicolumn{3}{|c|}{$\mathrm{NI}_{54}$} & \multicolumn{3}{|c|}{$N I_{\pi i \pi}$} \\
\hline Hp & da & $\mathrm{Hv}$ & $\mathrm{Hp}$ & $\mathrm{da}$ & $\theta \mathrm{v}$ & Hp & $\mathrm{da}$ & $0 \mathrm{v}$ & $\mathrm{Hp}$ & $\mathrm{da}$ & $0 \mathrm{v}$ \\
\hline 9,37 & 1,15 & 10,78 & 10,41 & 1,15 & 11,97 & 9,76 & 1,15 & 11,22 & 9,70 & 1,15 & 11,16 \\
\hline 9,38 & 1,20 & $1,1,26$ & 10.14 & 1,20 & 12,17 & 9,40 & 1,20 & 11,28 & 9.35 & 1,20 & 12.10 \\
\hline 9,44 & 1,20 & 11,33 & 9,30 & 1,20 & 11,16 & 11,62 & 1,20 & 13,94 & 10,62 & 1.20 & 12,74 \\
\hline 10,12 & 1,30 & 13.16 & $11,4]$ & 1,30 & 14,83 & 12,01 & 1,30 & $15.6 \mathrm{I}$ & 10,08 & 1.30 & 13,10 \\
\hline 12,69 & 1,35 & 17,13 & 12,31 & 1,35 & 16.62 & 12,43 & 1,35 & 16,78 & 14,81 & 1,35 & 19,99 \\
\hline 11,87 & 1,35 & 16,02 & 13,73 & 1,35 & 18.54 & 13,25 & 1,35 & 17,89 & 12,78 & 1,35 & 17,25 \\
\hline 12,32 & 1,35 & 16,63 & 13.96 & 1,35 & 18,85 & 14,06 & 1,35 & 18.98 & 13,84 & 1.35 & 18,68 \\
\hline 11,22 & 1,35 & 15,15 & 15,80 & 1,35 & 21,33 & 13,94 & 1,35 & 18,82 & 14,16 & 1,35 & 19,12 \\
\hline$(2,9)$ & 1,35 & 17,48 & 15.56 & 1,35 & 21,01 & 13,99 & 1,35 & 18.89 & 15,14 & 1,35 & 20,44 \\
\hline 13,43 & 1.35 & 18.13 & $15, ! 1$ & 1,35 & 20,40 & 14,45 & 1,35 & 19.51 & 13,83 & 1,35 & 18,67 \\
\hline 14.84 & 1,35 & 25,43 & 14,70 & 1,35 & 25,43 & 14,70 & 1.35 & 19,85 & $1 \cdot 4,83$ & 1,35 & 20,02 \\
\hline 14,96 & 1.35 & 20.20 & 14,13 & 1,35 & 19,08 & 13,84 & 1.35 & 18.68 & 13,19 & 1,35 & 17,81 \\
\hline 15,21 & 1.35 & 20,53 & 14,66 & 1,35 & 19,79 & 15,25 & 1,35 & 20,59 & 15,22 & 1,35 & 20,55 \\
\hline 15,27 & 1,35 & 20,61 & 14.58 & 1,35 & 19,68 & 14,55 & 1.35 & 19,64 & 16,84 & 1,35 & 22,73 \\
\hline 15,28 & 1,35 & 20,63 & 14,96 & 1,35 & 20,20 & 14,96 & 1,35 & 20,20 & 17,38 & 1,35 & 23,46 \\
\hline 15,00 & 1,35 & 20,25 & 13,90 & 1,35 & 18.77 & 12,15 & 1,35 & 16,40 & 21,21 & 1,35 & 28,63 \\
\hline
\end{tabular}


Les couvertures pédologiques à DVL comportent des horizons supérieurs microagrégés, à porosité visible forte, épais de plus d'un mètre et demi, passant progressivement en profondeur à des horizons à porosité plus faible, d'aspect compact, qui, sur pegmatite, sont constitués de matériatix d'altération à structure plus ou moins conservée.

Les zones à DVB dérivent des zones à DVL par une transformation liée à un abaissement relatif du niveau de base attribué à un léger soulèvement du socle guyanais (Boulet et al., 1979).

Le rôle important de ces deux types de drainage interne du sol est apparu globalement à l'échelle de l'hectare (bassin versant) ou de l'are (parcelle d'érosion). Ainsi l'écoulement des bassins est-il étroitement relié au pourcentage de la surface à DVL (Fritsch, 1981). De même SARRAilh (1981) met en évidence des différences de ruissellement très impottantes entre ces deux types de couvertures pédologiques (ruissellement annuel de 0,6 à 1,4 p. 100 de la pluic incidente en DVL et de 15 à 22 p. 100 en DVB).

Mais dans ces systèmes, l'étude détaillée de la dynamique de l'eau à l'échelle du profil, c'est-à-dire celle de l'interaction entre le cycle hydrologique dans le sol et les végétaux, n'a été qu'amorcéc (Humbel, 1978). Pour une telle étude notre choix s'est porté sur une zone de "basculement du drainage » car elle apparaissait comme un site clé où les deux dynamiques pouvaient être saisies et le passage de l'une à l'autre précisé.

\subsection{Le site cxpérimental}

La figure 3 constitue une coupe topo-pédologique schématisée du transect d'étude situé légèrement en contrcbas du sommet d'interfluve du bassin I et suivant une ligne de plus forte pente. Ce transect est caractérisé d'amont (DVL) en aval (DVB) d'une part par un amincissement progressif (incision de la surface topographique initiale), puis la disparition d'une couverture pédologique de type ferrallitique bien structuréc et à forte porosité interagrégats (horizons 1,2 et 3), d'autre part par la remontée progressive d'un matériau sablo-limono-argileux (horizon 6) présentant une structure d'altération de la roche-mère (pegmatite) de densité apparente plus élevée $(1,65$; cf. tabl. 1) et d'aspect plus compact. Les horizons de transition sableux (5) et sablo-limoneux (10) ont une densité apparente voisine de celle de l'horizon 6. Dans la suite, nous désignerons par "matériau d'altération" l'ensemble des horizons 6 , 5 et 10 .

La composition floristique, ainsi que la structure de la forêt primaire de la zone d'étude ont été étudiées par Puig (1979) et Lescure (1981). La densité du peuplement végétal est en moycnne de 630 individus (diamètre $>6 \mathrm{~cm}$ ) par hectare. Les Lécythidacées (Eschweilera spp), Cacsalpinacées (Eperua spp, Macrolobium sp) et Euphorbiacées sont les familles les plus représentées avec près de 50 p. 100 de l'effectif total de diamètre supéricur à $6 \mathrm{~cm}$. 


\subsection{Méthodes è techniques d'étude}

\subsection{Mesures hydriques in situ}

- En mai 1980, 5 batteries de tensiomètres ( $\mathrm{TI}_{1}$ à $\mathrm{TI}_{.}$) ont été installées en 5 sites ( $I_{1}$ à $\left.I_{-}\right)$du transect d'étude (fig. 3) pour l'établissement de profils verticaux du potentiel hydrique total $\left(\mathrm{T}_{\mathrm{t}}\right) \mathrm{du}$ sol. Les profondeurs de mesure étaient 10 , $20,40,60,80,100,120$ et $150 \mathrm{~cm}$.

- En août 1980, ont été mis en place 13 tubes d'accès neutronique en aluminium (diamètre intérieur $41 \mathrm{~mm}$ ), dont $2 \mathrm{en} I_{1}\left(N_{11}\right.$ et $\left.\mathrm{NI}_{12}\right), 2$ en. $I_{2}\left(\mathrm{NI}_{21}\right.$ et $\left.\mathrm{NI}_{2.2}\right)$, 2 en $I_{3,}, 2$ en $I_{4}$ et 5 en $I_{5}\left(\mathrm{NI}_{51}\right.$ à $\left.\mathrm{NI}_{5,5}\right)$ pour la mesure de l'humidité volumique du sol avec un humidimètre neutronique SOLO 20. Les mesures ont été effectuées tous les $10 \mathrm{~cm}$ entre 15 et $165 \mathrm{~cm}$ de profondeur.

- En janvier 1981, une batterie de tensiomètres supplémentaires ( $\mathrm{TI}_{0}$ ) a été installée en aval de $I_{1}$.

Dans la mesure du possible, les mesures neutroniques ont été effectuées une fois par semaine entre août 1980 et avril 1981. Afin de corriger les effets de dérive propre de l'électronique, toutes les valeurs de comptages neutroniques $\mathrm{N}$ ont été rapportées à des valeurs $\mathrm{N}_{\text {" }}$ de comptage-eau de référence établies avant et après les mesures dans les tubes d'accès. Les relevés tensiométriques ont été réalisés généralement deux ou trois fois par semaine de mai à fin septembre 1980 (date de désamorçage des tensiomètres), puis de janvier à juin 1981. La petite saison sèche de marsavril 1981 a fait l'objet d'un suivi plus serré destiné à l'étude des cinétiques de dessèchement, puis de réhumectation du sol.

\subsection{Etalonnage de l'humidimètre neutronique}

Pour l'établissement des relations d'étalonnage linéaires reliant l'humidité volumique $\theta\left(\mathrm{cm}^{3} / \mathrm{cm}^{3}\right)$ du sol au comptage neutronique standard $\left(\mathrm{N} / \mathrm{N}_{0}\right), 7$ échantillons provenant d'horizons divers ont été analysés pour l'établissement de leurs caractéristiques neutroniques au Centre d'Etudes Nucléaires de Cadarache, France (Covchat, 1977). Les résultats de cette analyse se présentent sous la forme $N / N_{0}=(\alpha d a+\beta) \theta$ $+\gamma \mathrm{da}+\delta$ où da est la densité apparente sèche du sol et $\alpha, \beta, \gamma$ et $\delta$ sont des coefficients d'étalonnage. Nous avons transformé cette équation en une équation du type $\theta=a \cdot N / N_{0}+b$ en prenant en considération les valeurs de da du tableau 1. Ces valeurs ont été obtenues par extrapolation de mesures faites au densitomètre à membrane le long du transect d'étude pédologique. Les équations ainsi obtenues sont rapportées par le tableau 2 .

La pente a de l'équation d'étalonnage reste relativement stable à l'intérieur de I'ensemble des échantillons issus des horizons $1,2,3,7$ et 8 (ensemble 1) (fig. 3) d'une part et des horizons 6 et 10 d'autre part (ensemble 2). Une différence sensible apparaît entre ces deux ensembles (en moyenne 94 pour l'ensemble 1, 80 pour l'ensemble 2). A l'intérieur de chacun de ces ensembles, l'ordonnée à l'origine b présente des variations plus importantes.

Nous avons confronté ces résultats à la « méthode de terrain» décrite par VALANCOGNE et al. (1977). 
J.M. GUEHL

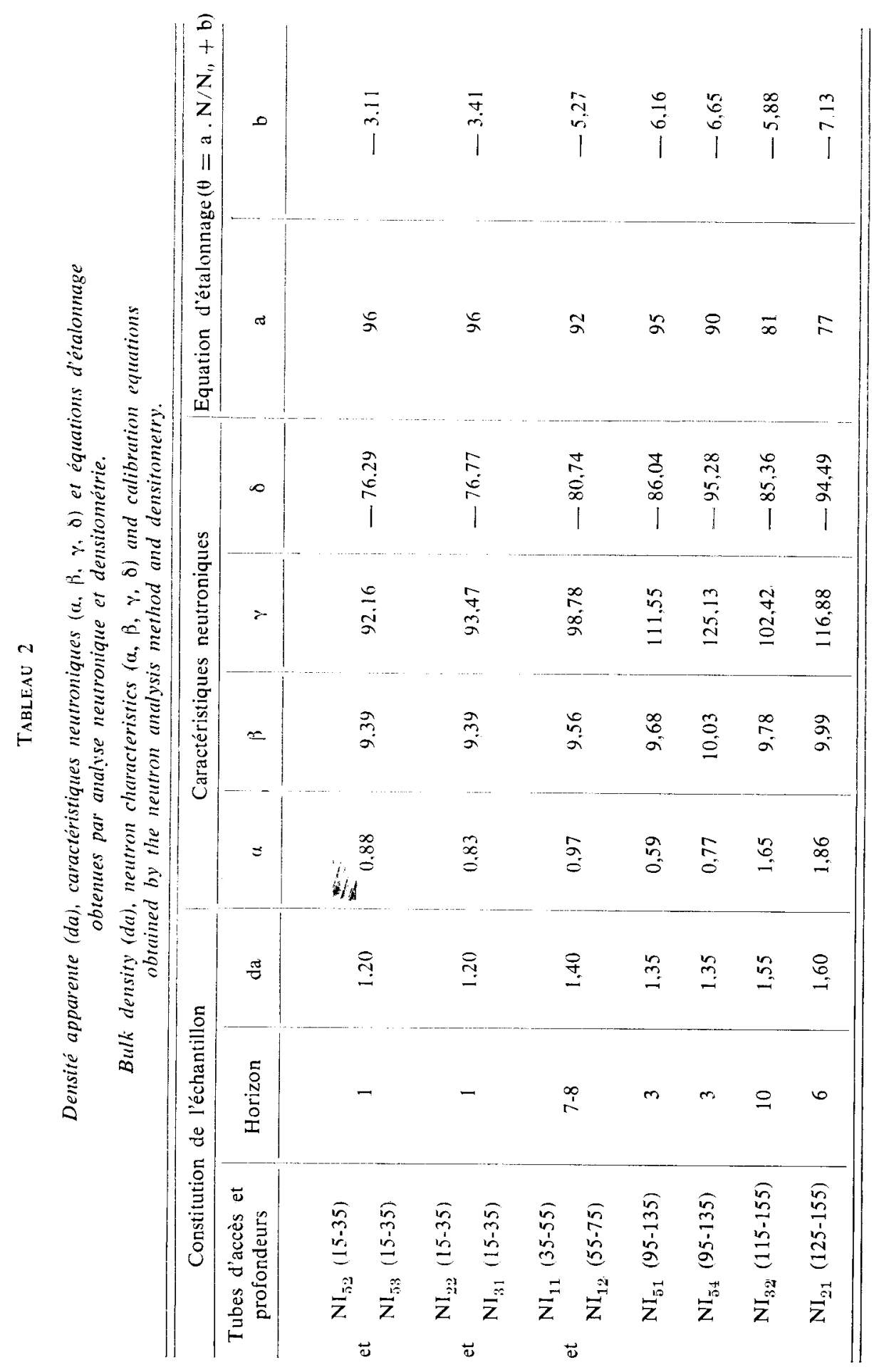




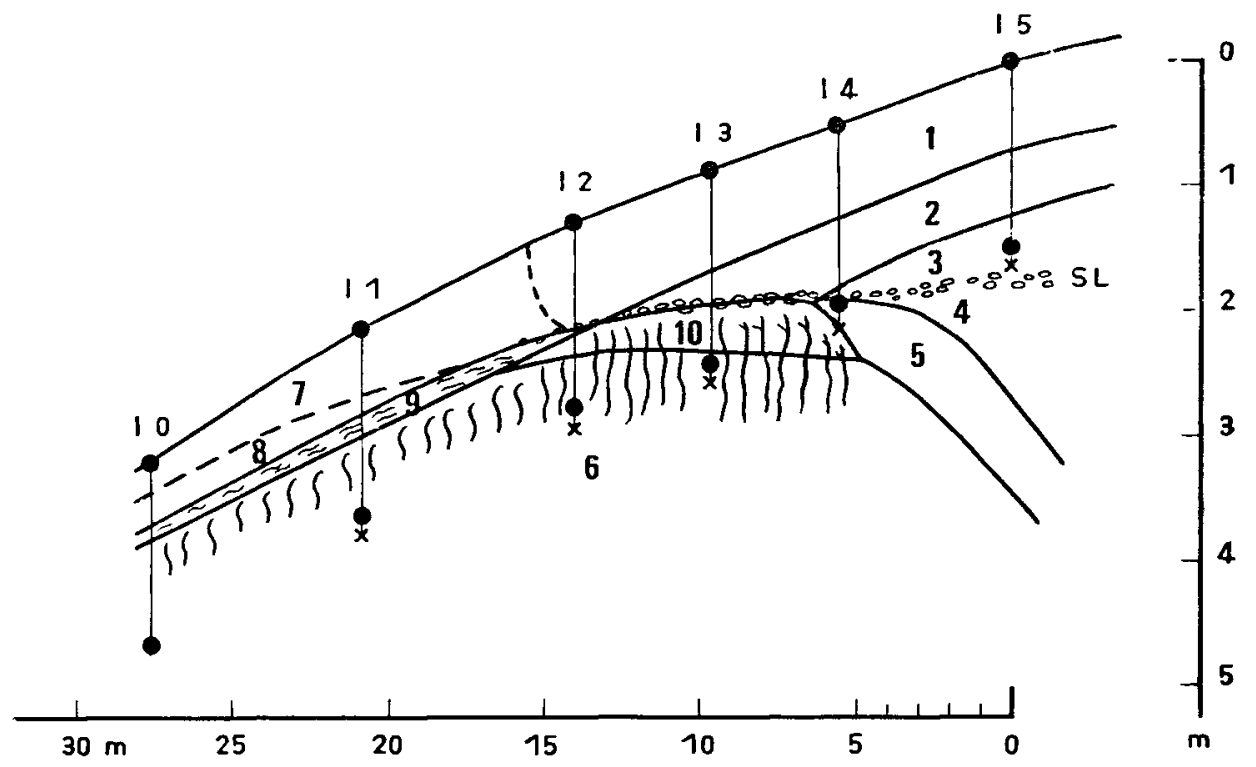

FIG. 3

Coupe topo-pédologique du transect d'étude. Les profondeurs des mesures tensiométriques et neutroniques $(X)$ sont indiquées en chacun des sites $I_{1 \prime}, \ldots, I_{. j}$.

Topographic and pedologic cut of the studied transect.

Tensiometric ( $\bullet$ and neutronic $(\times)$ measurement depths are represented for each site $I_{i t}, \ldots, I_{j}$.

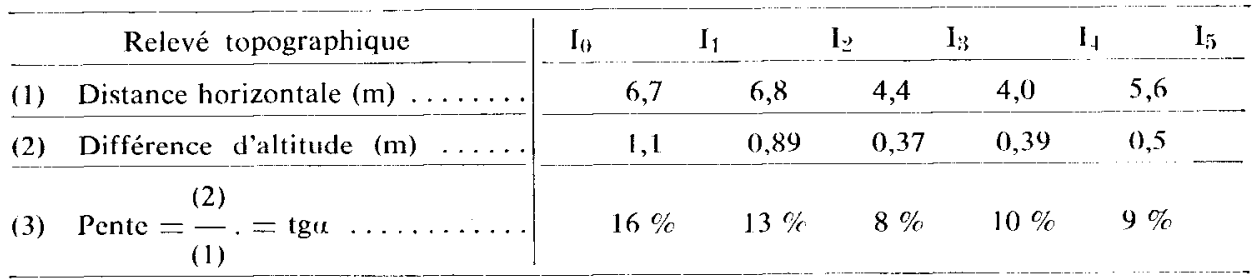

Description pédologique (d“après $\mathrm{R}$. Boulft et $\mathrm{Y}$ Luc'As) :

1: humifère, puis sablo-argileux, brun jaune à microagrégats.

2 : argilo-sableux à microagrégats.

3: argileux à microagrégats, rouge.

4 : sableux, riche en muscovite, rouge.

5 : sableux, brun-clair à volumes rouges.

6 : sablo-limono-argileux, blanc à volumes rouges,

faces structurales verticales lissées : \$SSS]

7 : ensemble humifère, puis sablo-argileux à agrégation biologique polyédrique, porosité tubulaire et interagrégats bien développée.

8 : brun jaune, sablo-argileux et argilo-sableux, porosité tubulaire bien développée.

9 : sableux à sable grossier. Chenaux millimétriques dans le sens de la pente : $\cong$

10: sableux jaune à volumes rouges, faces verticales moyennement lissées ou couvertes d’argile.

SL : Stone line. 
Cette méthode consiste en la détermination, avec des échantillons provenant de la mise en place des tubes d'accès, d'une part de l'humidité volumique (gravimétrie et densitométrie) établie par tranches de sol de $30 \mathrm{~cm}$ d'épaisseur (ordre de grandeur du diamètre de la sphère d'influence neutronique) et d'autre part des comptages neutroniques juste après installation des tubes. On tente alors de déduire les relations d'étalonnage de la représentation graphique de l'ensemble des points expérimentaux ainsi obtenus pour un horizon pédologique donné. Dans la présente étude, un tel ensemble de points a été obtenu lors de l'installation de tubes en août 1980, un second en avril 1981 dans une situation d'humidité du sol plus importante, grâce à l'installation passagère d'un tube à proximité de certains tubes en place.

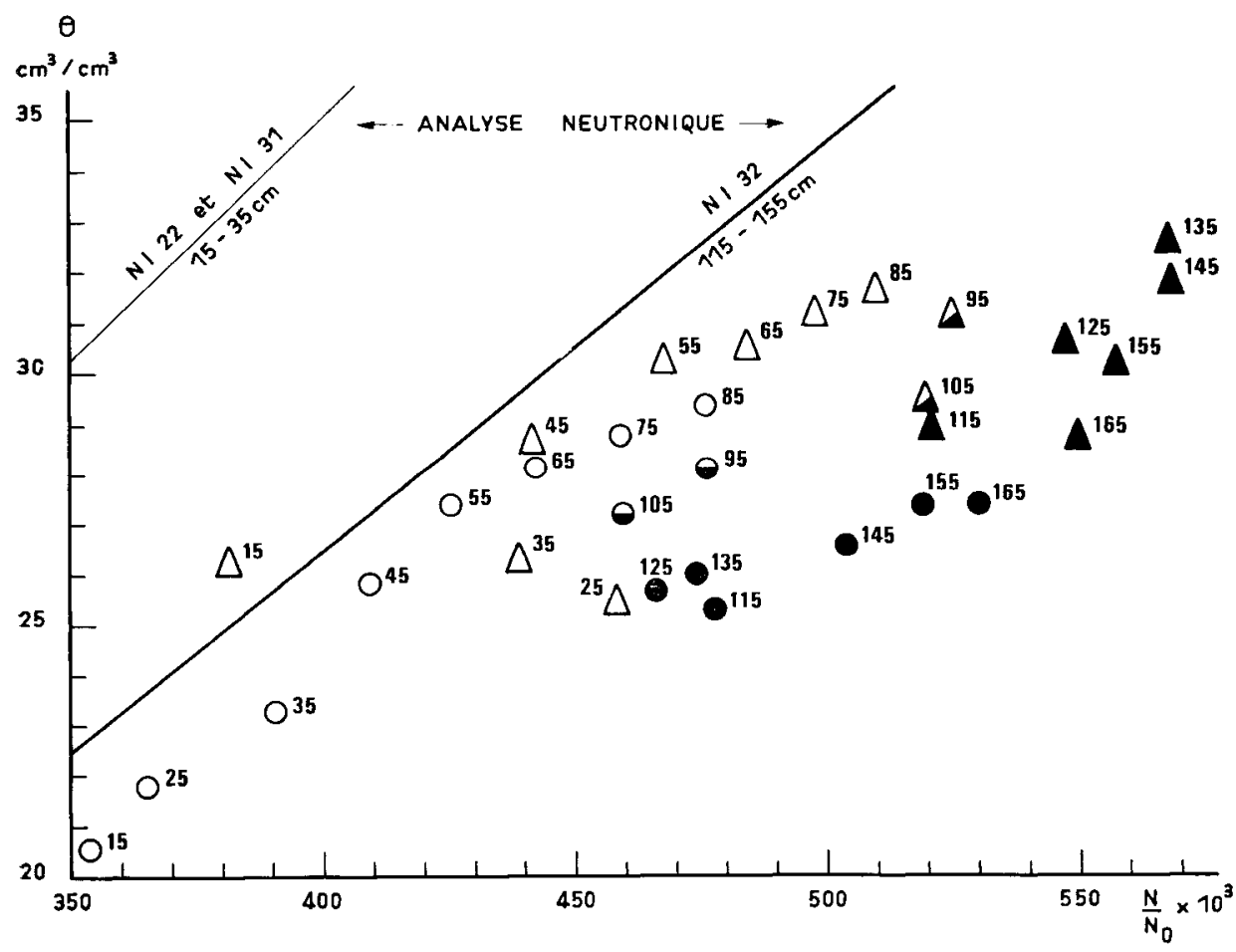

FIG. 4

Etalonnage de l'humidimètre neutronique. Comparaison de la méthode d'analyse neutronique et de la méthode de terrain. Tube $\mathrm{NI}_{32}$.

Calibration of the neutron-probe, comparison of the neutron analysis method and the field method for the neutron tube $\mathrm{NI}_{32}$.

Horizons 1 et $2:(O)$ août 1980, $\triangle$ ) avrıl 1981.

Niveaux de transition: (O) aout 1980, ( $\triangle$ ) avril 1981.

Horizons 10 et $6:(\bullet)$ août $1980,(\Lambda)$ avril 1981. 
De façon générale, la représentation graphique, tube par tube, de l'ensemble des points obtenus par la méthode de terrain fait apparaitre une nette discrimination entre les ensembles 1 et 2 décrits dans ce même paragraphe avec distinction d'un horizon intermédiaire d'épaisscur d"environ $20 \mathrm{~cm}$ (fig. 4). Cela est bien en accord avec les données du tableau 2.

En raison de la trop faible gamme dhumidité couverte par les points expérimentaux, la méthode de terrain ne permet toutefois pas ici une détermination indépendante précise de a et $b$. On notera toutcfois sur la figure 4 un accord satisfaisant cntre les deux méthodes pour ce qui concerne a et une nette surestimation de () par la méthode d'analyse neutronique. Ce cas de figure s'applique à l'ensemble des tubes.

En définitive, à l'instar de CosAndiy (1978), nous avons adopté une solution mixte en adoptant les valeurs de a fournies par la méthode d'analyse neutronique (94 pour l'ensemble 1 plus l'horizon 9, 80 pour l'ensemble 2 et 87 pour l'ensemble intermédiaire) (fig. 4) et en déterminant l'ordonnéc à l'origine b par calage des droites d'étalonnage sur chacun dés points expérimentaux de terrain obtenus en août 1980.

\subsection{Mesures d'infiltration anec l'appareil de Muntz}

Deux cylindres concentriques de dimensions standard sont enfoncés dans le sol sur une profondeur de $60 \mathrm{~mm}$. A l'aide de vases de Mariolte, on maintient une charge constante dans les cylindres. Le cylindre central, sur lequel on mesure l'évolution dans le temps de la quantité d'eau infiltrée par unité de surface $Q(t)$, est entouré d'un cylindre concentrique servant d'anneau de garde afin d'éviter les écoulements latéraux au niveau du cylindre central.

En milieu homogène un tel écoulement tend vers un régime permanent défini par l'équation simple (Hillel, 1974 ; Hallairl, communication personnelle) :

$$
\frac{d Q(t)}{d t}=K_{s}
$$

où $K_{*}$ est un coefficient traduisant la permćabilité du milieu et constituant une approximation par défaut de la conductivité hydraulique à saturation (cf. 2.1.) dans la direction verticale. Ce paramètre permet un classement des sols selon leur perméabilité dans des conditions standardisées simples.

\section{Résultats}

\subsection{Propriétés physiques du sol}

\subsection{Estimation de la perméabilité (Méthode de Muntz)}

Trois profils verlicatix de $K_{s}$ ont été établis par rafrâtchissement de fosses d'observations pédologiques. Ils apparaissent sur la figure 5 en relation avec la succession des horizons pédologiques. Il est possible d’inscrire chacun de ces 3 profils dans la coupe de la figure 3 : le profil A apparaît comme représentatif du site $I_{\ldots}$. B du site $I_{2 .}$ et $\mathrm{C}$ du site $I_{1}$. 


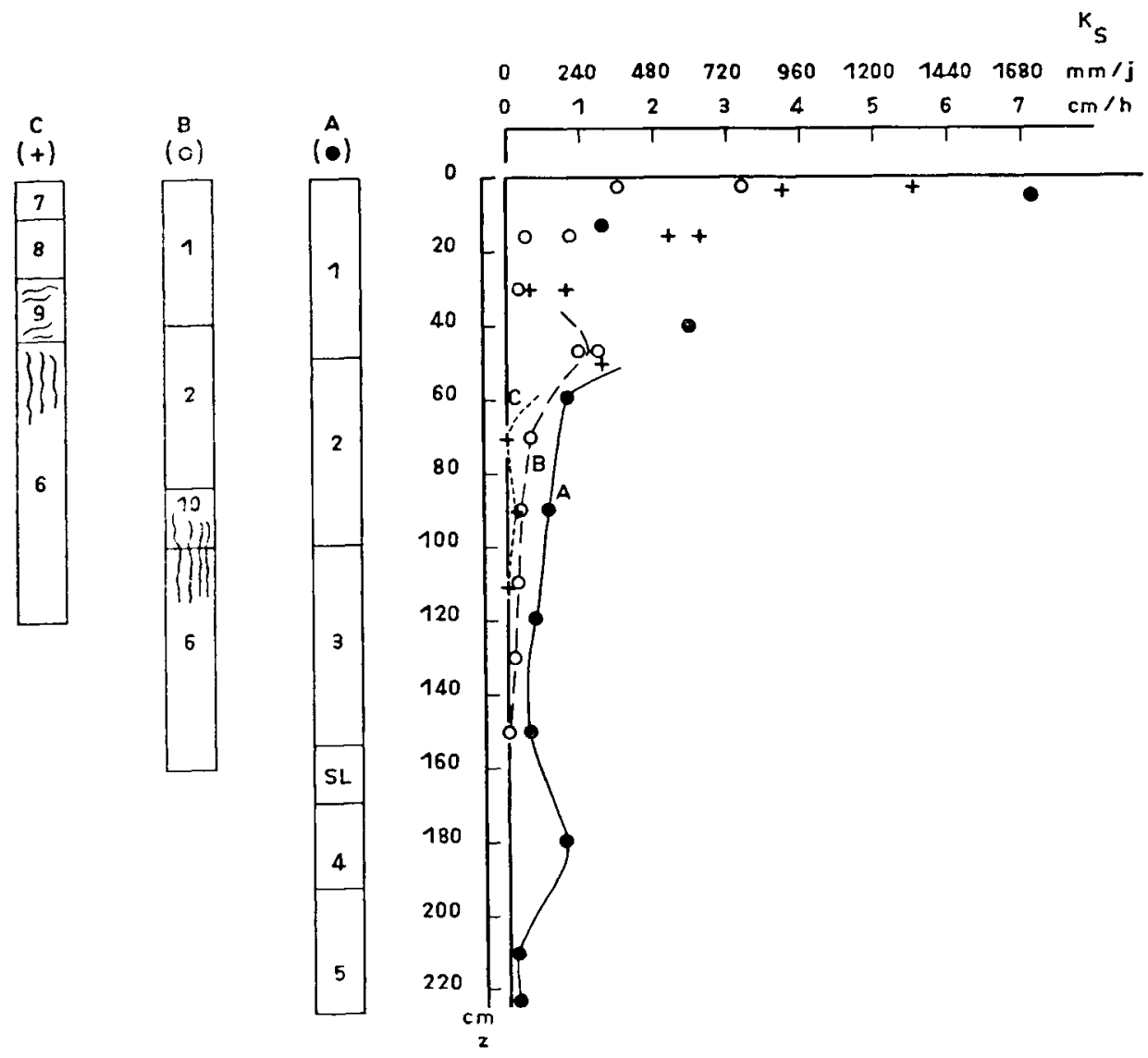

FIi. 5

Profils verticaur du coefficient $K_{s}$ (Méthode de Muntz).

Vertical profiles of $K_{x}$ (Muntz method) as related to the pedologic organization.

Chenaux millimétriques.

5 SIS Faces verticales.

Dans le cas du profil $A$, on nole une diminution importante de $K_{\text {. entre } 0 \text { et }}$ $60 \mathrm{~cm}$. Cette diminution se poursuit d'une façon moins marquée jusquà des valeurs voisines de $3 \mathrm{~mm} / \mathrm{h}$ à la base de l'horizon argileux 3. Des valeurs plus importantes de l'ordre de $8 \mathrm{~mm} / \mathrm{h}$ sont notées dans l'horizon 4 et au sommet de l'horizon 5 sableux à sable grossier, $K_{*}$ rediminuant ensuite $(1 \mathrm{~mm} / \mathrm{h})$ à l'approche de l'horizon 6 .

Les valeurs de $K_{s}$ ont également élé exprimées en $\mathrm{mm} / \mathrm{jour}$, cela permet une comparaison directe avec les valeurs pluviométriques. $K$. correspond en effet pratiquement à la quantité maximale d'eau que le sol peut absorber sans provoquer un engorgement au-dessus du niveauu considéré (ZASLAVSK1, 1964). Cette comparaison ne 
s'applique toutefois qu'au voisinage de la surface, puisqu'en profondeur les flux sont retardés et atténués par un terme de mise en réserve dans les horizons sus-jacents.

Les faibles valeurs de $K_{*}$ notées dans le cas des profils $B$ et $C$, à moins de $40 \mathrm{~cm}$ de profondeur, peuvent, en saison des pluies et lors des épisodes orageux de début et de fin de grande saison sèche. entrainer de tels engorgements temporaires de surface (pour les valeurs de pluviométrie, on se reportera aux données de la figure 12). Dans le cas de ces deux derniers profils, la réaugmentation de $K_{s}$ à $50 \mathrm{~cm}$ de profondeur pourrait être attribuée au réseau de faces verticales caractérisant le somriet de l'horizon 6 en prenant en considération le fait que la mesure caractérise en réalité une zone sous-jacente à l'appareil.

L'enfoncement dans l'horizon blanc à volumes rouges (6) s'accompagne d'une diminution continue de $K_{s}$, aucun écoulement vertical n'est plus enregistré dès $70 \mathrm{~cm}$ de profondeur pour le profil $C$ et dès $150 \mathrm{~cm}$ pour le profil $B$, malgré la poursuite des mesures durant toute une nuit. La valeur non nulle observée à $90 \mathrm{~cm}$ de profondeur pour le profil $\mathrm{C}$ semble cependant prouver dans ce matériau l'existence de possibilités locales d'écoulement vertical.

Les différences de $K_{x}$ apparaissant pour les niveaux profonds entre le profil $A$ (au-dessus de la Stone-line) d'une part et les profils B et C d'autre part, sont bien en accord avec les différences de densité apparente (tabl. 1) indiquant une différence de porosité totale.

En effet la porosité totale $p$ est reliée à da par la relation :

$$
\mathrm{p}=1-\frac{\mathrm{da}}{\mathrm{dr}} \simeq \mathrm{I}-\frac{\mathrm{da}}{2.65}
$$

où dr est la densité réelle du sol $(\mathrm{dr} \simeq 2.65)$.

Pour les horizons profonds, on a donc :

- cas de l'horizon $3(\mathrm{da} \simeq 1,35)=\mathrm{p} \simeq 49$ p. 100 ;

- cas de l'horizon $6(\mathrm{da} \simeq 1.65)=\mathrm{p} \simeq 38 \mathrm{p} .100$.

Mais, outre la porosité totale. la distribution des pores par classes de diamètre est un facteur déterminant de lá conductivité hydraulique du sol. Au voisinage de la saturation, notamment, on sait que les valeurs élevées de conductivité hydraulique sont subordonnées à l'existence de pores de diamètre élevé (macroporosité de diamètre supérieur à 8 f(m) (Brutsaert, 1964 ; JaIl.LARD, 1980).

\subsection{Courbe caractéristique de l'humidité du sol $\mathrm{Y}_{\mathrm{m}}(\mathbf{( 1 )}$. Importance et répartition de la macroporosité}

La relation $\Psi_{m}(\theta)$ est soumise à un effet d'hystérésis se traduisant par l'existence de courbes différentes suivant l'état hydrique du sol et suivant le sens des variations d'humidité (sorption ou désorption), (Hil.L.LL, 1974 : Vachaud et al., 1978).

A partir des données tensiométriques et neutroniques, nous avons établi des courbes $\Psi_{\mathrm{m}}(\theta)$ in situ en phase de désorption au cours de la petite saison sèche de mars 1981 et au début de la grande saison sèche de septembre 1980. Trois exemples de relations $I_{m}(0)$ sont représentés par la ligure 6 (ajustement graphique). Outre leur 
signification hydrodynamique propre, ces courbes permettent une bonne estimation de la répartition de la macroporosité du sol (JAILI.ARD, 1981).

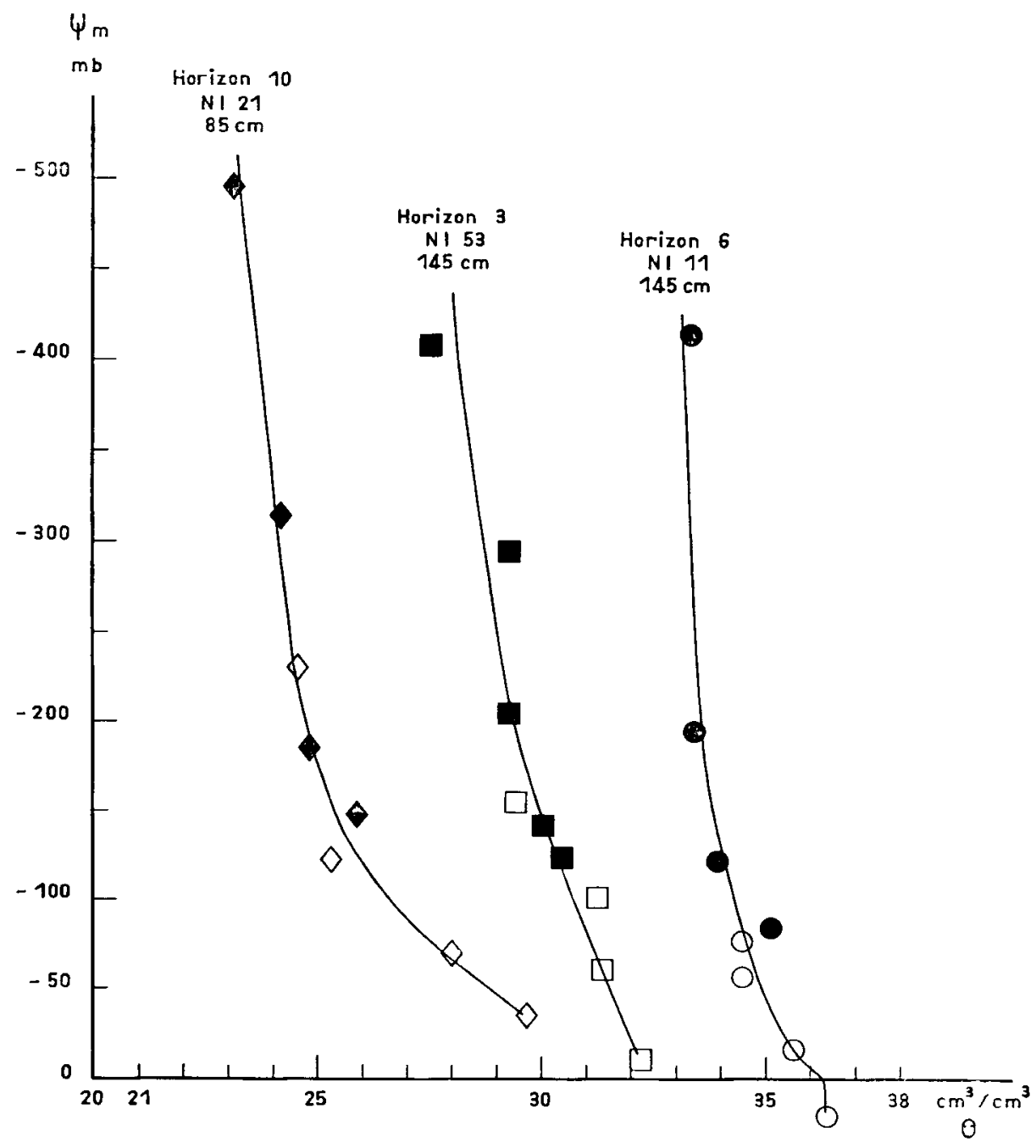

FIG. 6

Courbe caractéristique de l'humidité du sol ' $\mathrm{I}_{{ }_{2}}$ (0) pour des niveaux de mesure appartenant aux horizons. 3,6 et 10.

Soil matric potential - soil water content relationship $\Psi^{\prime \prime}(\theta)$ for measurement levels of the horizons 3, 6 and 10.

En effet, entre 0 et -1 bar, le potentiel matriciel est surtout lié à la rétention capillaire (HiLlel, 1974). Si l'on admet que les pores sont cylindriques et que l'angle de contact eau-sol est nul, ' ${ }^{\prime}{ }_{11}$ est relié au diamètre $D$ des plus gros pores non encore vidangés par l'équation : 


$$
-\Psi_{\mathrm{m}}=\frac{4 \sigma}{\mathrm{D}}
$$

où $\sigma$ est la tension superficielle à l'interface eau-air $\left(\sigma=0,072 \mathrm{~kg} \mathrm{~s}^{-2} \simeq 720 \mathrm{mbar}\right.$ (um) à $25^{\circ} \mathrm{C}$.

Si l'on suppose de plus qu'à saturation l'ensemble des pores sont remplis d'eau et que la désorption se fait par vidange totale et successive des pores suivant leur diamètre décroissant, à une diminution du potentiel matriciel de $\Psi_{m_{4}}$ à $\Psi_{m_{2}}$ correspond une diminution d'humidité volumique égale au volume des pores de diamètre

compris entre $D_{1}=-\frac{4 \sigma}{\Psi_{m 1}}$ et $D_{z}=-\frac{4 \sigma}{\Psi_{n z}}$.

Ces hypothèses n'étant jamais totalement vérifićes dans la réalité. notamment en raison de la complexité de la forme des pores et de leurs interconnexions, cette estimation doit être considérée comme un moyen de classification des sols plutôt que comme une approche des valeurs réelles.

La figure 7 fait apparaître la répartition de la macroporosité pour différents horizons accessibles aux appareils de mesure. Les bornes des classes de diamètre correspondent aux valeurs suivantes de $\mathrm{I}_{\mathrm{m}}$ :

\begin{tabular}{c|c|c|c|c|c|c|c|c|c}
\hline $\begin{array}{c}\mathrm{D} \\
\left(\mathrm{Am}_{\mathrm{m}}\right)\end{array}$ & 4,8 & 5,8 & 7,2 & 9,6 & 14,4 & 28,8 & 57,6 & 115 & $x$ \\
\hline $\begin{array}{c}\mathrm{M}_{\mathrm{m}} \\
(\mathrm{mb})\end{array}$ & -600 & -500 & -400 & -300 & -200 & -100 & -50 & $\cdots 25$ & 0 \\
\hline
\end{tabular}

La non existence de points expérimentaux au voisinage de la saturation ne pernet pas l'estimation de la macroporosité de taille supérieure à 115 flm dans le cas des horizons $3,7,9$ et 10 et supérieure à $57,6 \mathrm{~m}$ dans le cas de l'horizon 2. Ces valeurs auraient probablement été très élevées puisque l'étude morphologique des horizons concernés révèle l'existence d'une porosité structurale de type polyédrique dans le cas des horizons 2 et 7 , de chenaux millimétriques pour l'horizon 9 et de fissures verticales pour l'horizon 10 .

Dans la partie aval du transect d'étude, la figure 7 (partie droite) met en évidence une opposition entre d'une part les horizons de surface 7 et 9 (sablo-argileux), 10 (sablo-limoneux) et 5 (sableaux) à forte macroporosité et d'autre part lhorizon 6 de macroporosité faible.

En $I_{5}$ (partic gauche de la figure 7), on notera que le passage de l'horizon 2 à l'horizon 3 argileux (kaolinite) s'accompagne d'une diminution importante de la macroporosité. 


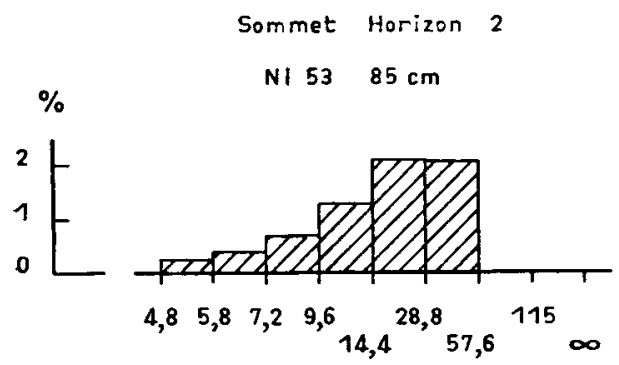

Horizon 3

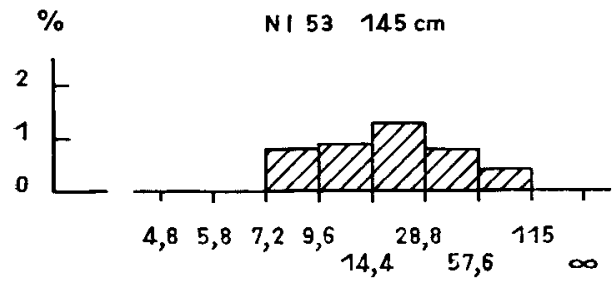

Sommet Horizon 5

NI $41145 \mathrm{~cm}$

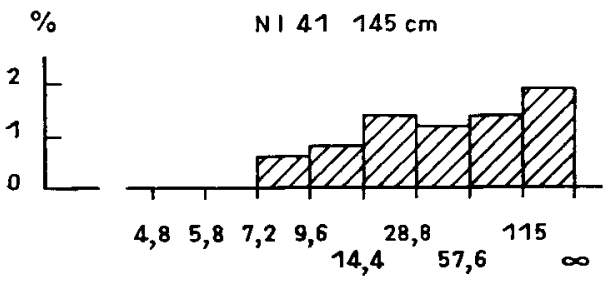

Base Horizon 7

NI $22 \quad 85 \mathrm{~cm}$

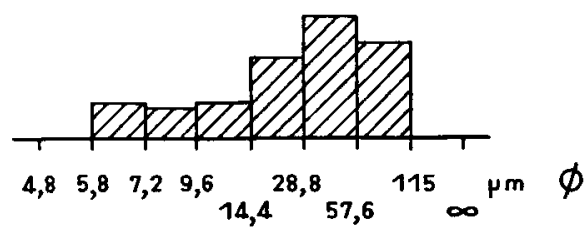

Horizon 9

NI $1165 \mathrm{~cm}$

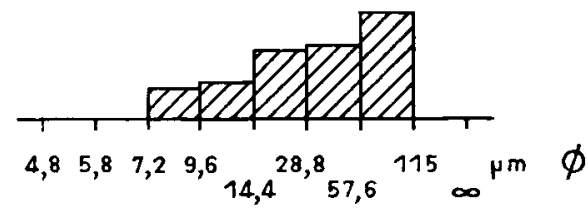

Horizon 10

NI $2185 \mathrm{~cm}$

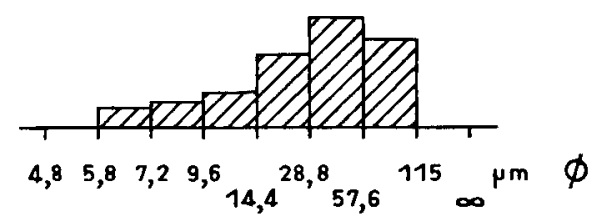

Horizon 6

NI $11145 \mathrm{~cm}$

15

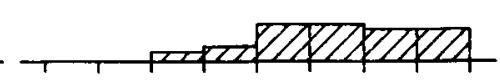

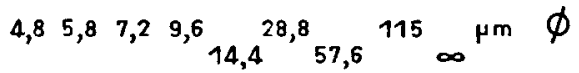

FIG. 7

Répartition de la macroporosité pour les principaux horizons du transect d'étude.

Pore-size distribution of the main horizons of the studied transect. 


\begin{tabular}{|c|c|c|c|c|c|c|c|c|c|c|c|c|c|}
\hline & 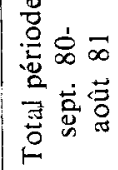 & $\begin{array}{l}0 \\
\vdots \\
a \\
\ddot{n}\end{array}$ & 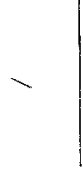 & $\begin{array}{c}E \\
E \\
m \\
\infty \\
+\end{array}$ & 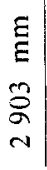 & \begin{tabular}{c|}
$E$ \\
$E$ \\
$n$ \\
$n$ \\
$\sim$ \\
-
\end{tabular} & $\gamma$ & $\begin{array}{l}E \\
\Xi \\
0 \\
\sigma \\
-\end{array}$ & $\begin{array}{l}E \\
E \\
n \\
n \\
-\end{array}$ & $\begin{array}{l}\varepsilon \\
\Xi \\
\infty \\
\infty \\
\infty \\
-\end{array}$ & \begin{tabular}{l|}
$E$ \\
$\Xi$ \\
$\infty$ \\
$\infty$ \\
$\infty$ \\
-
\end{tabular} & $\begin{array}{c}\varepsilon \\
\Xi \\
\tilde{\sigma} \\
\tilde{n} \\
=\end{array}$ & $\begin{array}{l}E \\
E \\
D \\
n \\
n \\
-\end{array}$ \\
\hline & 䓂 & $\vec{\sim}$ & $\begin{array}{l}g \\
\dot{f}\end{array}$ & $\begin{array}{l}n \\
\dot{f} \\
\dot{y}\end{array}$ & $\stackrel{\overbrace{}}{二}$ & $\stackrel{g}{2}$ & $\stackrel{\Xi}{\Xi}$ & む゙ & $\Xi$ & $\frac{0}{1}$ & $\frac{1}{1}$ & ț & 号 \\
\hline & $\bar{\Xi}$ & $\ddot{\sim}$ & $\stackrel{m}{i}$ & $\begin{array}{l}\infty \\
m \\
m\end{array}$ & $\frac{m}{m}$ & $\underset{⿱}{త}$ & $\stackrel{2}{=}$ & 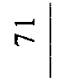 & 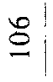 & $\infty$ & $\widehat{\stackrel{\sim}{v}}$ & 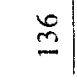 & $\stackrel{\uplus}{\beth}$ \\
\hline$\dot{\Xi}$ &.$\Xi$ & $\underset{\sim}{*}$ & $\begin{array}{l}\infty \\
\dot{g} \\
\dot{q}\end{array}$ & $\begin{array}{l}\overrightarrow{0} \\
\dot{v}\end{array}$ & $\stackrel{\infty}{m}$ & $\stackrel{\varrho}{\Xi}$ & $\stackrel{\infty}{\approx}$ & in & $\infty$ & $\overrightarrow{\hat{\imath}} \mid$ & $\stackrel{3}{3}$ & $\Xi$ & $\underset{\infty}{+}$ \\
\hline 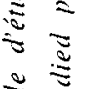 & $\bar{\pi}$ & $\stackrel{\vartheta}{\sigma}$ & $\stackrel{y}{q}$ & $\begin{array}{c}- \\
\infty \\
\infty\end{array}$ & $\underset{\mathcal{Y}}{\tilde{y}}$ & $\vec{m}$ & ஜ & in & $\infty$ & $\vec{a}$ & 志 & ম & $\infty$ \\
\hline 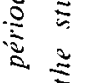 & $\dot{3}$ & $\tilde{w}_{0}^{m}$ & $\frac{\theta}{\dot{t}}$ & $\frac{\dot{m}}{m}$ & $\frac{\vec{m}}{m}$ & $\underline{m}$ & ジ & 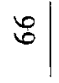 & $\infty$ & $\underset{\infty}{ \pm}$ & $\underset{\sim}{2}$ & $\stackrel{\vec{g}}{\Xi}$ & $\tilde{\beth}$ \\
\hline 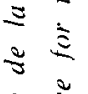 & $\sum^{\infty}$ & 2 & $\begin{array}{l}n \\
i n\end{array}$ & $\begin{array}{l}m \\
m \\
m\end{array}$ & 8 & $\underset{\Xi}{J}$ & $\bar{\beth}$ & $\cong$ & 6 & in & 5 & $\begin{array}{l}0 \\
0 \\
2\end{array}$ & 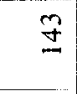 \\
\hline$\underset{\Xi}{\Xi} \underset{\Xi}{\Xi}$ & 㐫 & $\stackrel{\sim}{i}$ & $\stackrel{\sim}{\tilde{m}}$ & $\begin{array}{l}m \\
\vec{n}\end{array}$ & $\frac{9}{\sigma}$ & 苍 & 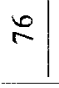 & $g$ & 2 & $\frac{n}{m}$ & $\stackrel{0}{\S}$ & 5 & $\stackrel{\infty}{a}$ \\
\hline$\stackrel{2}{ \pm}$ & 莣 & in & $\ddot{g}$ & $\begin{array}{l}\infty \\
\dot{v} \\
\dot{y}\end{array}$ & $\stackrel{?}{\sim}$ & 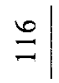 & 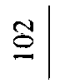 & $\hat{\sigma}$ & $\stackrel{2}{2}$ & $\stackrel{+}{2}$ & $m$ & 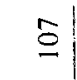 & 8 \\
\hline$\stackrel{\Xi}{\Xi}$ & ڤ̊ & m & $\vec{f}$ & $\frac{a}{m}$ & $\stackrel{\vec{\sim}}{\sim}$ & $\stackrel{0}{=}$ & $\S$ & 6 & 8 & \begin{tabular}{l}
0 \\
\hdashline
\end{tabular} & $\underline{\sim}$ & $\cong$ & $\stackrel{m}{\Xi}$ \\
\hline ミี & z & $\ddot{n}$ & 9 & $\underset{8}{\mathscr{y}}$ & $\overrightarrow{0}$ & $\begin{array}{l}\mathscr{0} \\
\simeq\end{array}$ & $\stackrel{8}{\circ}$ & 8 & 昌 & $m$ & $\simeq$ & $\stackrel{n}{2}$ & q \\
\hline$\Xi \lesssim$ & $\dot{\overrightarrow{0}}$ & $\vec{\oplus}$ & $\begin{array}{l}m \\
\infty \\
\infty \\
n\end{array}$ & in & $\stackrel{0}{=}$ & $\begin{array}{l}\infty \\
m\end{array}$ & 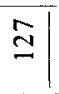 & $\stackrel{ \pm}{\simeq}$ & $\infty$ & $\stackrel{N}{N}$ & $\begin{array}{c}\hat{b} \\
\mid\end{array}$ & $\infty$ & $\Xi$ \\
\hline 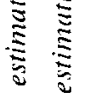 & 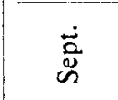 & बें & - & 8 & Dי & $\stackrel{m}{\Xi}$ & - & $\stackrel{0}{\simeq}$ & $\begin{array}{l}\infty \\
\infty \\
\infty\end{array}$ & $\frac{5}{1}$ & $\frac{n}{n}$ & $\underline{6}$ & $\widetilde{\Xi}$ \\
\hline 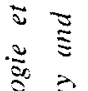 & 蒿 & $\underset{0}{\forall}$ & - & $\vec{n}$ & $\stackrel{9}{=}$ & - & & & & & & & \\
\hline 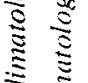 & $\ddot{\Xi}$ & $\stackrel{\infty}{\sim}$ & - & $\underset{\approx}{\approx}$ & $\stackrel{\wp}{\dddot{m}}$ & - & & & & & & & \\
\hline & & 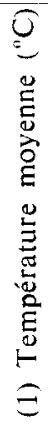 & 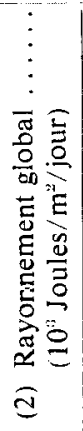 & 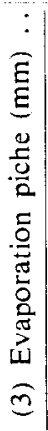 & 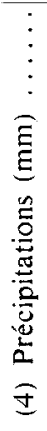 & 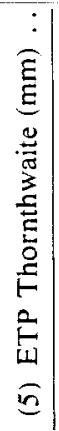 & 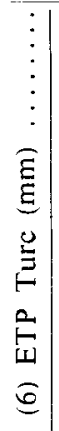 & 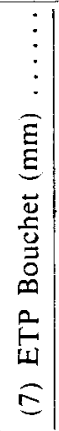 & 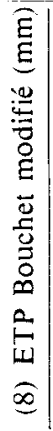 & 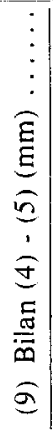 & 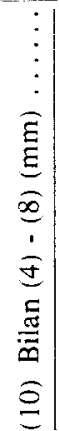 & 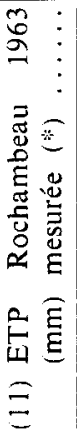 & 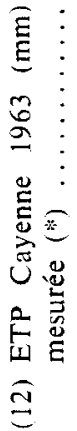 \\
\hline
\end{tabular}




\subsection{Dynamique de l'eau dans le sol en conditions naturelles}

\subsection{Climatologie de la période étudiée}

A partir des données climatologiques (tabl. 3) fournies par un poste (abri Météorologie Nationale de type classique) que nous avions installé à $500 \mathrm{~m}$ environ du bassin 1 (parcelle Arbocel) dans une situation de coupe rase, il est possible d'estimer l'évapotranspiration potentielle (E.T.P.) par les formules de Thornthwaite (température de l'air), Turc (rayonnement global et température de l'air) et Botuchet (évapotranspiration Piche et température de l'air) (Brochet \& Gerbier, 1975).

Cette dernière formule s'écrit : E.T.P. $=a[1+\lambda(\mathrm{T})]$ Ep où Ep représente l'évaporation Piche $(\mathrm{mm}), \lambda(\mathrm{T})$ une fonction connue de la température de laair et « un coefficient dépendant à la fois des conditions de mesure (abri, évaporomètre, installation) et de paramètres climatiques régionaux.

Sur la base des travaux de Fougriouze (1966) menés dans la région de Cayenne |zone plus côtière que notre site, pour la zonation climatique de la Guyane, on pourra consulter Fougerouzs: (1965)l, nous avons adopté une valeur $\alpha=0.5$.

La valeur annuclle d'E.T.P. Thornthwaite obtenuce $(1515 \mathrm{~mm})$ est une valeur vraisemblable pour la Guyane (MADEc, 1963). Par contre, les méthodes de Turc et Bouchet fournissent des valeurs nettement trop faibles. Concernant la formule de Bouchet, ce résultat est en désaccord avec les conclusions de Fougerouzı: (1966) ayant retenu cette formule comme une estimation satisfaisante de l'E.T.P. à l'échelle mensuelle. Parmi lęs facteurs de variation de ú évoqués ci-dessus, le fait que notre abri ait été installé dans une situation de trouée dans la parcelle Arbocel (diamètre de la trouée : environ $60 \mathrm{~m}$, hauteu du recrú à l'alentour : $8 \mathrm{~m}$ ) où la ventilation est probablement inféricure à celle régnant atl-dessus de la forêt, nous semble constituer l'explication la plus vraisemblable de cette différence.

La considération des bilans menstels P.E.T.P. Thornthwaite met en évidence l'existence de deux périodes de déficit hydrique (E.T.P. $>$ P) :

- septembre-octobre 1980 (grande saison sèche, déficit $=129 \mathrm{~mm}$ );

- mai 1981 (petite saison sèche, déficit $=54 \mathrm{~mm}$ ).

Ces valeurs de déficit constituent en fait une sous-estimation de la réalité : en effet, dans les situations climatiques du type de celle de la Guyane de faible amplitude thermique annuelle (tabl. 3), la formule de Thornthwaite ne prenant en compte que la seule composante thermique du elimat ne rend compte que de façon amortie des variations saisonnières de l'E.T.P.

Une situation plus proche de la réalité est sans doute celle obtenue par considération dans le bilan hydrique d'une E.T.P. Bouchet multipliée par un facteur correctif E.T.P. Thornthwaite

Égal at rapport annuel : $=1,49$, aboutissant à des déficits E.T.P. Bouchet

de 221 et $97 \mathrm{~mm}$ pour les deux périodes de déficit. On notera que les valeurs obtenues par cette formule de Bouchet modifiée fournissent des valeurs très voisines de celles trouvées par Fouglrouzi: (1966) par la formule de Bouchet à Rochambeau et Cayenne dans les situations extrêmes et bien typées de septembre-octobre (grande saison sèche) et de mai-juin (saison des pluies). 
Le tableau 3 fait en outre apparaître un bilan largement excédentaire de décembre 1980 à février 1981 et de mai à juillet 1981.

\subsection{Etude de l'écoulement souterrain en situation d'excès d'eau}

\subsection{Analyse des profils $\mathrm{\Psi}_{\mathrm{t}}(\mathrm{z})$ le long du iransect d'étude}

La figure 8 présente les 6 profils verticaux du potentiel hydrique total obtenus le 23 février 1981 lors d'une période fortement pluvieuse. L'altitude de référence $\left(\Psi_{\mathrm{g}}=0\right)$ est prise à la surface du sol en chacun des sites, la représentation graphique ainsi obtenue permet une comparaison inter sites de l'état de l'eau et de la nature des flux :

- $I_{5}$ : Drainage vertical en milieu non saturé suivant un gradient de potentiel sensiblement unitaire (écoulement gravitaire en milieu relativement homogène).

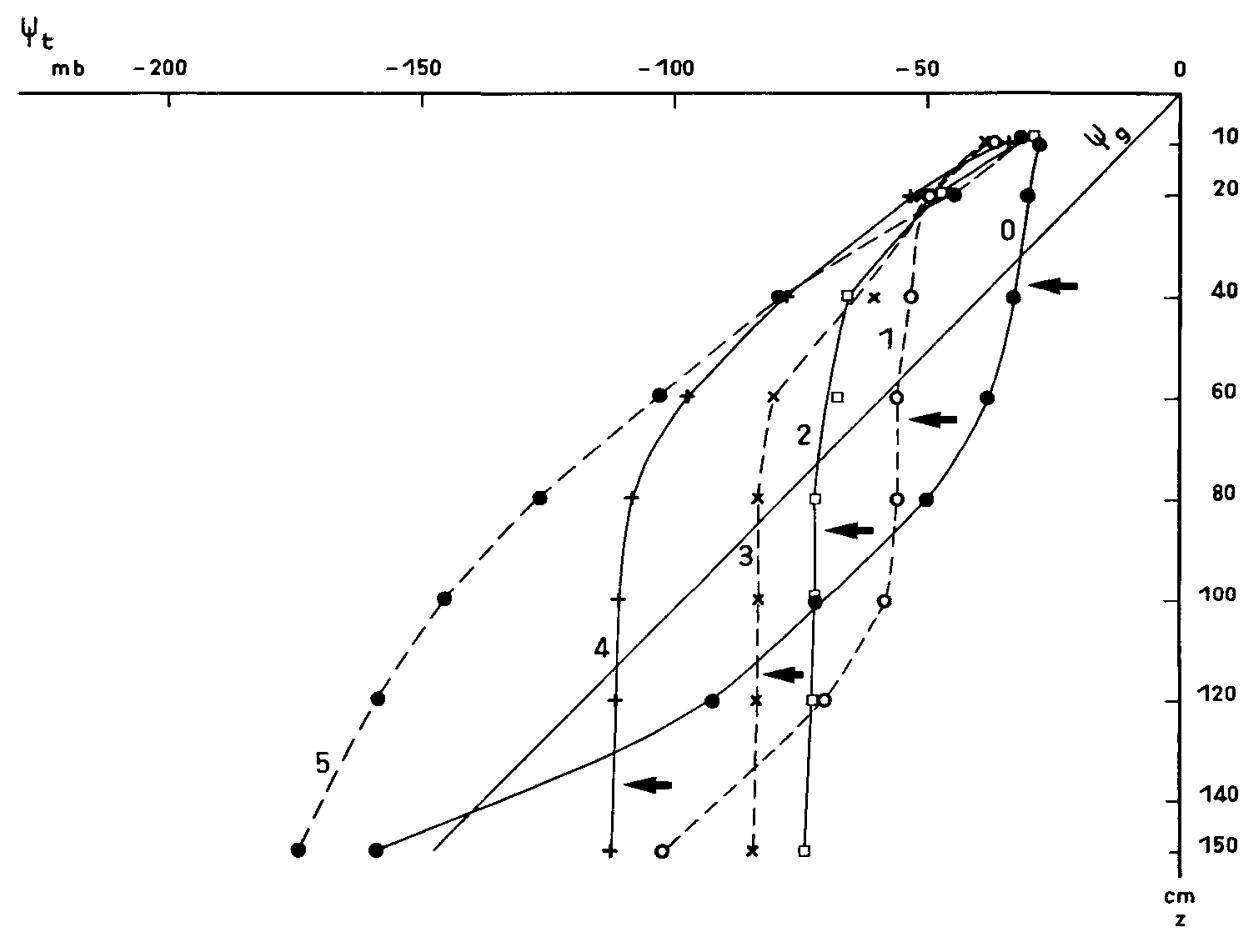

FIG. 8

Profils verticaux du potentiel hydrique total ( $\left.\Psi_{\text {, }}\right)$ le long du transect d'étude en situation d'excès d'eau (23 février 1981).

$\Psi_{g}$ est amnulé à la surface du sol en chacun des sites. $0: T I_{b}, \ldots, 5: T I_{i j}$.

Les flèches indiquent la profondeur d'apparition du matériau d'altération de la pegmatite.

Vertical profiles of the total soil water potential $\left(\Psi_{f}\right)$ along the studied transect for a water excess situation (February 23th 1981).

$\Psi_{g}$ is set to 0 at the soil surface for each of the sites. $0: T I_{0,}, \ldots, 5: T I_{5}$.

The arrow's indicate the depth of appearance of the altered rock material (Pegmatite). 
- $I_{4}, I_{3}$ et $I_{2 .}$ : Apparition d'une nappe d'eau à $z_{11}$ à $113 \mathrm{~cm}$ en $I_{4}, z_{11}=85 \mathrm{~cm}$ en $I_{3}$ et $z_{I I}=75 \mathrm{~cm}$ en $I_{2 .}$. La remontée du niveau du toit de la nappe de $I_{4}$ à $I_{2 .}$ (se poursuivant d'ailleurs pour $I_{1}$ et $I_{11}$ ) est bien cohérente avec la remontée du niveau d'apparition du matériau d'altération. L'inexistence de perte de charge perceptible verticalement dans la nappe indique d'une part un écoulement vertical nul ou très faible et d'autre part que le plancher de la nappe (zone de très faible conductivité hydraulique) est situé à plus de $150 \mathrm{~cm}$ de profondeur.

Les profils observés en $I_{1}$ et $I_{0}$ sont plus complexes, mais peuvent être interprétés à la lumière des travaux de ZASI.AvSKI (1964) portant sur le régime permanent de l'infiltration en milieu saturé dans le cas de profils hétérogènes (modèles pluricouches avec contrastes de perméabilité). L'hypothèse de régime permanent (écoulement limité par les caractéristiques du sol) est très vraiscmblablement vérifiée ici puisque la figure 8 caractérise une situation oblenue après plusieurs jours de pluies importantes (fig. 12).

- $I_{1}$ : Toit de la nappe à $z_{11}=55 \mathrm{~cm}$ de la surface. On note une perte de charge dans le domaine hydrostatique à partir de $80 \mathrm{~cm}$ de profondeur, soil $20 \mathrm{~cm}$ environ sous le niveau d'apparition du matériau d'altération (horizon 6). Cela indique une diminution importante de $K_{s}$ à ce niveau. Entre 120 et $150 \mathrm{~cm}$ de profondeur cette perte de charge est de gradient unitaire (milieu localement homogène). Le flux de drainage vertical est alors numériquement égal à la conductivité hydraulique à saturation $K_{s}$ |équation (6)| et contrôle l'écoulement vertical de la nappe (rôle limitant des zones de plus faible conductivité). $K_{*}$ étant très faible (fig. 5), le flux vertical est également très faible (matériau-plancher de la nappe).

- $I_{0}$ : Jusqu'à $100 \mathrm{~cm}$ de profondeur, situation qualitativement identique à la tranche $0-150 \mathrm{~cm}$ de $I_{1}$. L'augmentation du gradient de $\Psi_{t}$ se poursuivant au-delà de $100 \mathrm{~cm}$ de profondeur et le retour dans $\mathrm{le}$ domaine $\Psi_{\mathrm{m}}<0$ à $140 \mathrm{~cm}$ indique une réaugmentation de $K_{*}$ légèrement sous le niveaul $150 \mathrm{~cm}$.

\subsection{Champ du potentiel hydrique total}

Dans cette partie, nous adoptons pour l'ensemble des sites de mesure une allitude de référence commune : la surface du sol en $I_{.}$. Cette convention permet une approche bi-dimensionnelle du fonctionnement hydrodynamique du système étudié suivant un axe vertical et l'axe du transect d'étude. Ce dernier suivant une ligne de plus forte pente, on peut en effet admettre que l'écoulement qui lui est normal (donc suivant les courbes de niveau) est nul.

Sur les figures 9 à 11 , nous avons représenté le champ du potentiel hydrique total sous la forme de lignes équipotentielles dans 3 situations différentes. Cette représentation repose sur une interpolation spatiale entre les sites de mesure.

Les points suivants sont à considérer pour l'interprétation des figures 9 à 11 :

- Pour permettre la représentation graphique de l'ensemble du système étudié, nous avons été contraints d'exagérer l'échelle verticale 5 fois par rapport à l'échelle horizontale. Cette transformation donne aux gradients horizontaux une importance 5 fois trop élevéc par rapport aux gradients verticaux. La verticalité et l'horizontalité des équipotentielles sont néanmoins conservées par cette distorsion. 


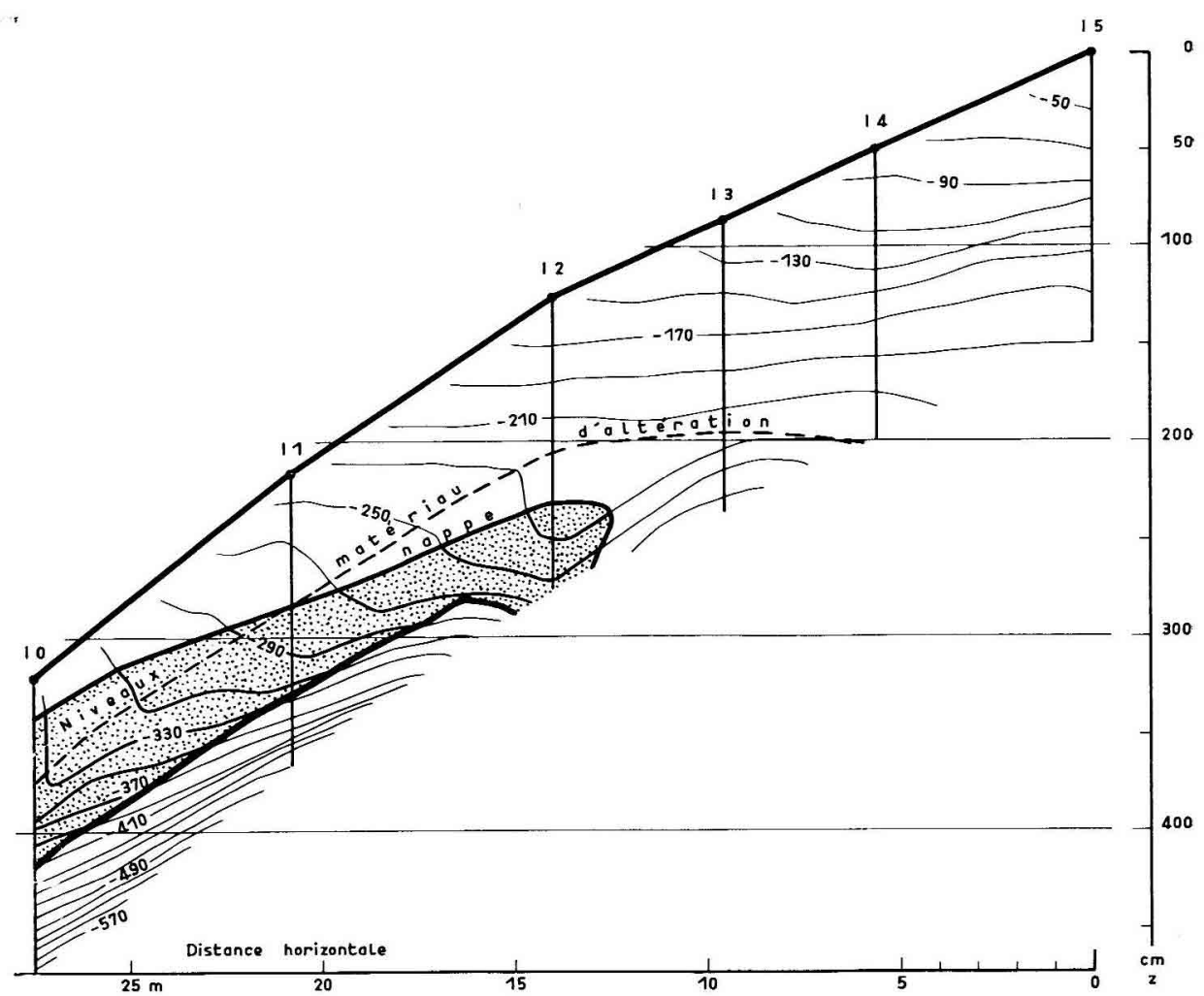

FIG. 9

Champ du potentiel hydrique total ( $\Psi_{t}$, courbes équipotentielles).

Situation d'apparition de la nappe perchée (15 avril 1981). $\Psi_{g}$ est annulé à la surface du sol en $\mathbf{I}_{5}$.

Two-dimensional field of the total water potential ( $\Psi_{/}$, equipotentials). Situation of appearance of the perched water table (April 15th 1981). $\Psi_{g}$ is set to $O$ at the soil surface of $I_{j}$.

En trait épais et zone pointillée: domaine hydrostatique $\left(\Psi_{m}>0\right)$.

En trait fin

Thick line and dotted area

Fine line

Thick broken line

domaine matriciel $\left(\Psi_{\mathrm{m}}<0\right)$.

hydrostatic field $\left(\Psi_{m}>0\right)$.

matric field $\left(\Psi_{m<0}<\right.$ ).

appearance of the altered rock material.

- Pour tout point des figures 9 à il, il est possibile de déterminer par simple projection graphique sur l'axe des profondeurs le potentiel de gravité (1) et par différence avec le potentiel total la composante matricielle ou hydrostatique $\left(\Psi_{m}\right) \mathrm{du}$ potentiel total. Cette opération simple permet notamment de délimiter graphiquement les domaines matriciel et hydrostatique (en trait gros et en pointillé sur les figures 9 à 11).

(1) A $100 \mathrm{~cm}$ de profondeur correspondent 98 millibars. 
- Dans un système hydrodynamique isotrope les flax sont normaux aux équipotentielles et dirigés dans le sens des potentiels décroissants [équation (7)]. Le lecteur pourra ainsi aisénent imaginer les lignes de courant correspondant dans l'hypothèse d'isotropie au système d'équipotentielles représenté. Si le milieu est anisotrope, la composante verticale du gradient du potentiel indique également l'existence d'une composante verticale du flux, la composante horizontale indique une composante horizontale du flux, mais la valeur du gradient ne permet pas d'apprécier immédiatement les grandeurs respectives de ces composantes et donc la direction exacte des flux par rapport aux équipotentielles.

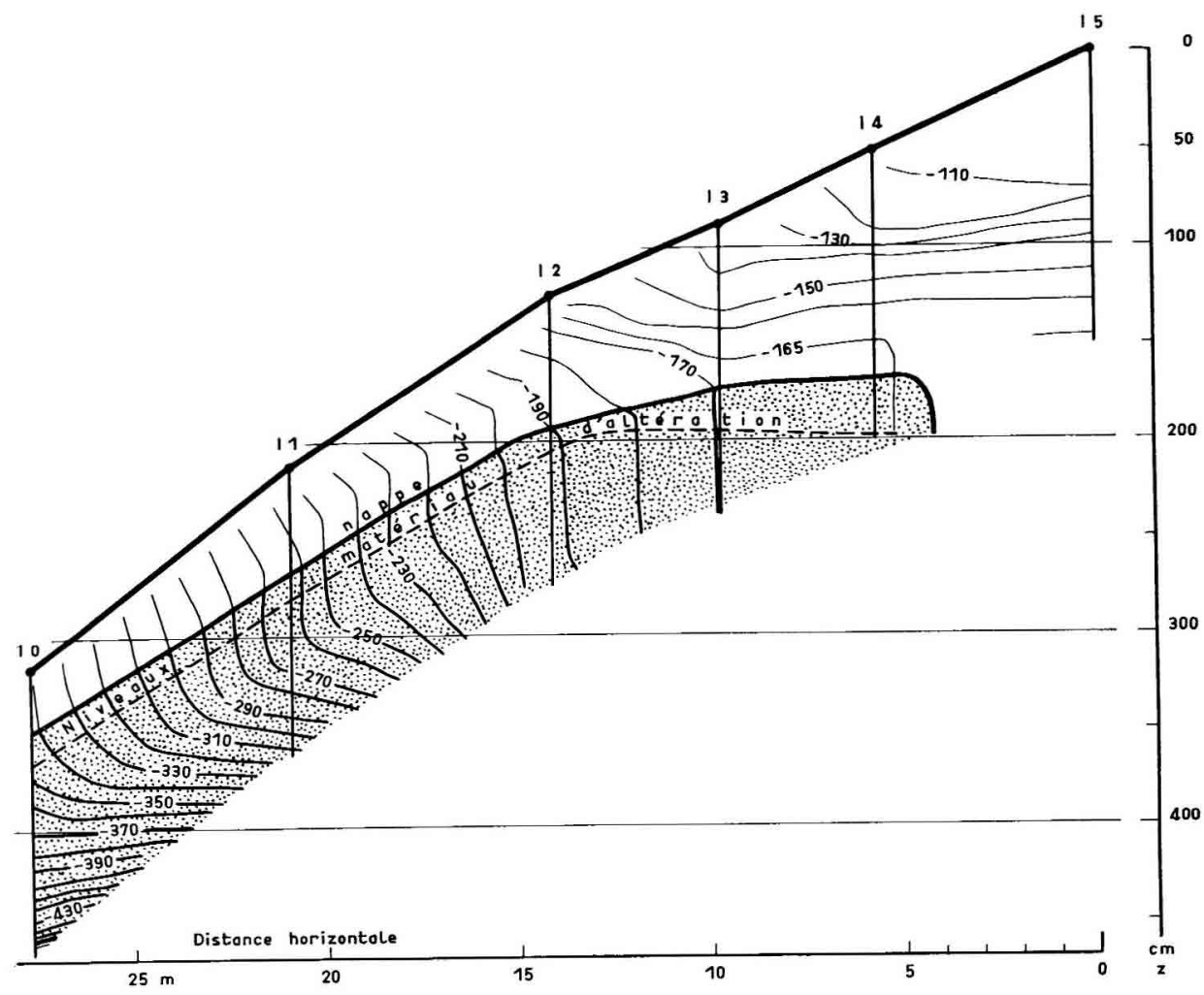

Fig. 10

Champ du potentiel hydrique total (w).

Situation d'extension maximale de la nappe perchée (23 février 1981). Mêmes conventions que figure 9.

Field of the total water potential $\left(\Psi_{t}\right)$.

Maximal development of the perched water table (February 23th 1981).

Same conventions as in figure 9.

La figure 9 présente une situation de réhumectation du sol (15 avril 1981) après la petite saison sèche de mars (pour les données pluviométriques se reporter à la figure 12). De $I_{5}$ à $I_{3}$, on note un écoulement vertical non saturé suivant un gradient 
sensiblement gravitaire (cf. courbe 5 de la figure 8), le profil est bien réhumecté sur toute son épaisseur. De $I_{2}$ à $I_{0}$, existe une zone de pression positive (nappe perchée) sous laquelle on observe une zone matricielle caractérisée par un gradient vertical de $\Psi_{t}$ très important. Cela traduit la progression vers le bas d'un front de réhumectation dans l'horizon 6 de très faible conductivité hydraulique. Dans la partie la plus superficielle du domaine hydrostatique et surtout en aval de $\mathrm{I}_{1}$ (horizons « bien structurés » 7,8 et 9) on note l'apparition d'une composante latérale de l'écoulement vers le bas de pente.

La figure 10 (23 février 1981) caractérise une période très pluvieuse, la nappe atteint ici son extension maximale en profondeur, le point de profondeur $150 \mathrm{~cm}$ en $I_{t)}$ restant dans le domaine matriciel (cf. 3.221.). On notera la très bonne coïncidence entre la variation spatiale du toit de la nappe et celle de la profondeur d'apparition du matériau d'altération et, ce qui est équivalent (Livet, 1976) entre le gradient latéral de $\Psi_{t}$ dans la nappe au voisinage de son sommet et la pente du matériau d'altération. Cela confirme le rôle de matériau plancher de la nappe joué par l'horizon 6. L'écoulement saturé est essentiellement latéral suivant la pente du matériau plancher, la zone de fort gradient vertical de $\Psi_{t}$ en milieu saturé apparaissant en aval de $I_{2}$ se situe en effet dans la zone de très faible conductivité hydraulique de l'horizon 6 (cf. 3.11.).

La figure 11 (25 mai 1981) présente une situation de retrait de la nappe au cours d'un épisode peu pluvieux de la saison des pluies. Le domaine hydrostatique n'existe plus qu'en profondeur dans l'horizon 6 et a totalement disparu des sites $I_{4}$ et $I_{3}$, ainsi que du site $I_{0}$. La disparition de la nappe de ce dernier site peut s'expliquer :

- soit par l'augmentation de la pente existant en aval de $I_{10}$;

- soit par l'existence d'un écoulement vertical non négligeable en $I_{i}$, cette hypothèse est bien en accord avec les remarques faites à propos de la courbe 0 au 3.221., ainsi qu'avec les observations de Boulet (1981) notant une réinversion du type de drainage en aval du présent système de différenciation pédologique latérale.

\subsection{Fluctuations du toit de la nappe}

L'existence d'une nappe et le niveau de son toit ont été déterminés d'après les relevés tensiométriques (fig. 2 et 8 ). Les résultats obtenus lors de 2 périodes de mesures (fin mai à fin septembre 1980, puis fin janvier à fin mai 1981) sont rapportés par la figure 12 , en relation avec le régime des précipitations. Le site $I_{0}$ n'existe que pour la seconde période d'étude. L'origine des profondeurs est la surface du sol en chacun des sites.

En $I_{\text {, }}$ on ne note à aucun moment présence de nappe.

Pour les sites $I_{1}$ à $I_{1}$, apparaît pour l'ensemble des relevés caractérisés par l'existence d'une nappe une diminution progressive de la profondeur du toit de la nappe, bien en accord avec la remontée vers la surface du matériau d'altération. Entre $I_{1}$ et $I_{11}$, cet ordre n'est pas respecté dans certaines situations de rabattement de la nappe (4 mars, 13 mai 1981 et 23 mai 1981). Des hypothèses explicatives de ces situations particulières ont été données au 3.222 . 


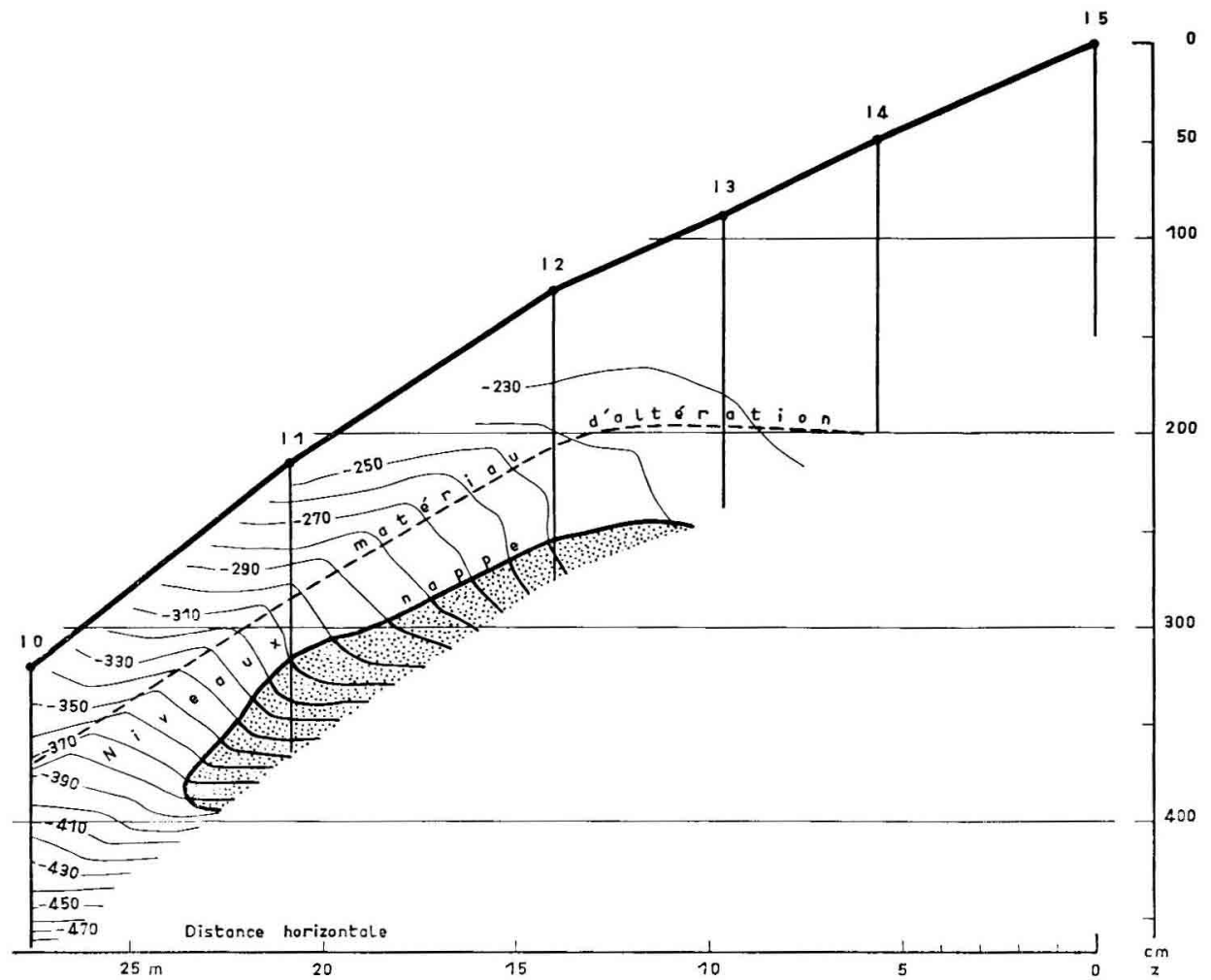

FiG. 11

Champ du potentiel hydrique total $\left(\Psi_{t}\right)$.

Situation de disparition de la nappe perchée (25 mai 1981). Mêmes conventions que figure 9.

Field of the total water potential $\left(\Psi_{1}\right)$.

Disappearance of the perched water table (May 25'l' 198I). Same conventions as in figure 9.

La réhumectation après la petite saison sèche de mars 1981 présente une image de réapparition de la nappe à partir d'une situation totalement hors saturation : la nappe apparait en $I_{6}$ et se développe ensuite progressivenent jusqu'en $I_{1}$. Cela traduit le rôle tampon croissant des horizons superficiels $7,8,9,1$ et 2 de porosité élevée en fonction de l'augmentation de leur épaisseur.

De façon générale, lors des épisodes peu pluvieux, on note un rabattement rapide de la nappe traduisant un drainage latéral efficace et une disparition rapide de la nappe dès le débul des deux saisons sèches sáns possibilité significative de contribution au bilan hydrique des périodes ultérieures caractérisées par un déficit hydrique climatique (tabl. 3). 

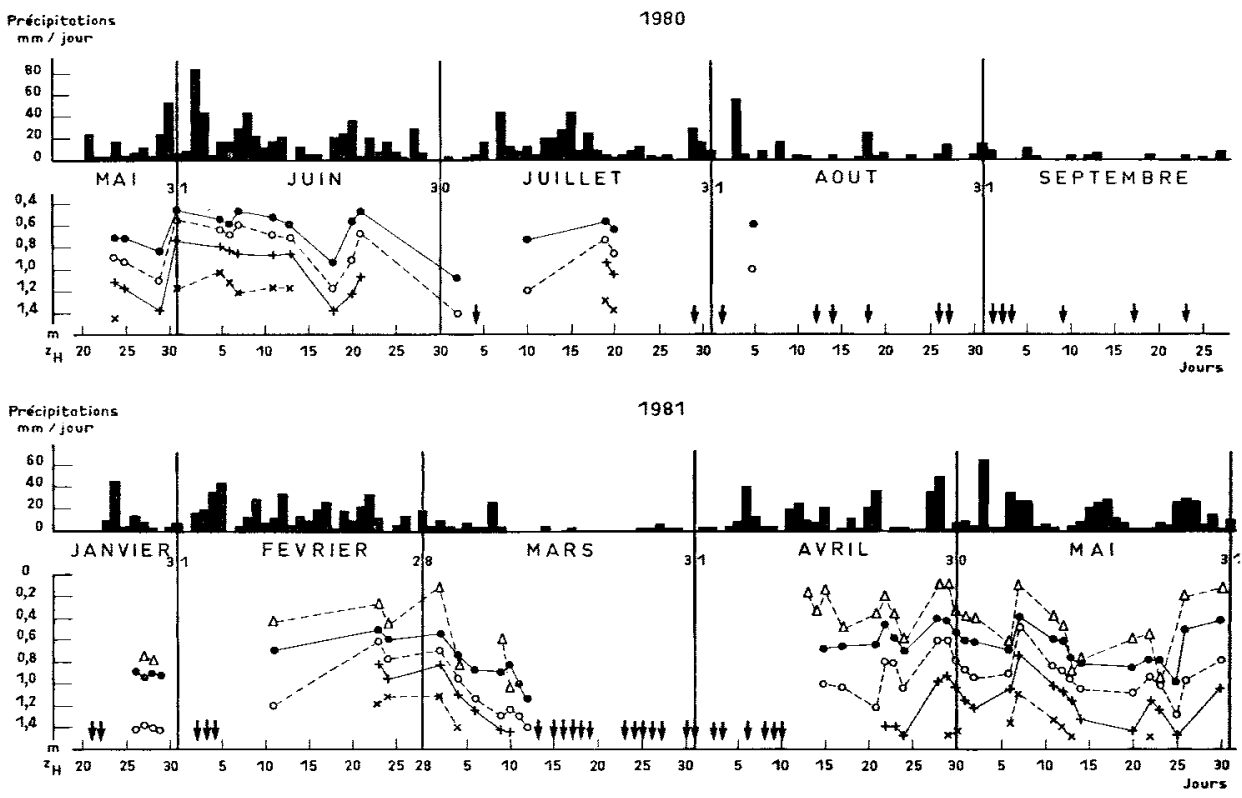

FIG. 12

Fluctuations du niveat de la nappe perchée par rapport à la surface da sol en In $(\triangle-\Delta)$, $I_{1}(\bullet-\bullet), I_{2}\left(\bigcirc_{-}\right), I_{3}(+-+)$ et $I_{4}(\times-\times)$ en relation avec le régime pluviométrique. Les flèches indiquent les dates de mesures tensiométriques effectuées en l'absence de nappe.

Fluctuations of the perched water table level (distance to the soil surface for each site) for $I_{11}(\triangle-\triangle), I_{1}(-\longrightarrow), I_{2}(\bigcirc-O), I_{3}(+-+)$ and $I_{4}(\times-\times)$ as related to the rainfall regime.

The arrows indicate the dates of tensiometric measurements made in the absence of water table.

\subsection{Evolution des profils hydriques et des flux hydriques profonds} lors des saisons sèches

Nous nous limiterons ici à l'examen de quelques profils particuliers de $\theta$ et de $\mathrm{Y}_{\mathrm{t}}$ dans la partic aval (fig. $13 \mathrm{~A}$ ) et amont (fig. $13 \mathrm{~B}$ ) du transect d'ótude.

- 6 et 15 mars 1981 : début de la petite saison sèche de 1981. Au 6 mars, on note encore la présence de la nappe en profondeur en $I_{1}$ et $I_{2}$.

- 30 mars 1981 : fin de la petite saison sèche.

- 23 et 30 septembre 1981 : profils obtenus au cœur de la grande saison sèche de 1980, durant l'ensemble du mois de septembre les précipitations journalières ne dépassent pas quelques millimètres.

- 5 novembre 1980 : profil caractérisant la fin de la grande saison sèche. C'est le profil le plus « sec » malgré l'occurrence de pluies orageuses importantes au cours du mois d'octobre (50 $\mathrm{mm}$ le 19 octobre). 
Le profil 7 correspond à l'humidité volumique au $\mathrm{pF} 4,2$ (valeurs «lissées» prenant en compte trois niveaux successifs de profondeur du tableau 1), paramètre classiquement considéré comme la borne inféricure de la réserve hydrique utile du sol. Concernant les valeurs du tableau 1 , on notera une nette diminution de l'humidité pondérale avec l'entrée dans l'horizon 6 , cependant que pour l'humidité volumique ces différences sont amoindries par les différences de densité apparente.

Bien que n'appartenant pas à une même suite chronologique, ces différentes situations n'en représentent pas moins un continuum de l'état de dessèchement du sol.

Aux cotes 120 et $150 \mathrm{~cm}$, les mesures tensiométriques étaient possibles durant l'ensemble de la petite saison sèche; durant la grande saison sèche, aucune mesure tensiométrique n'était plus possible à partir du 30 septembre.

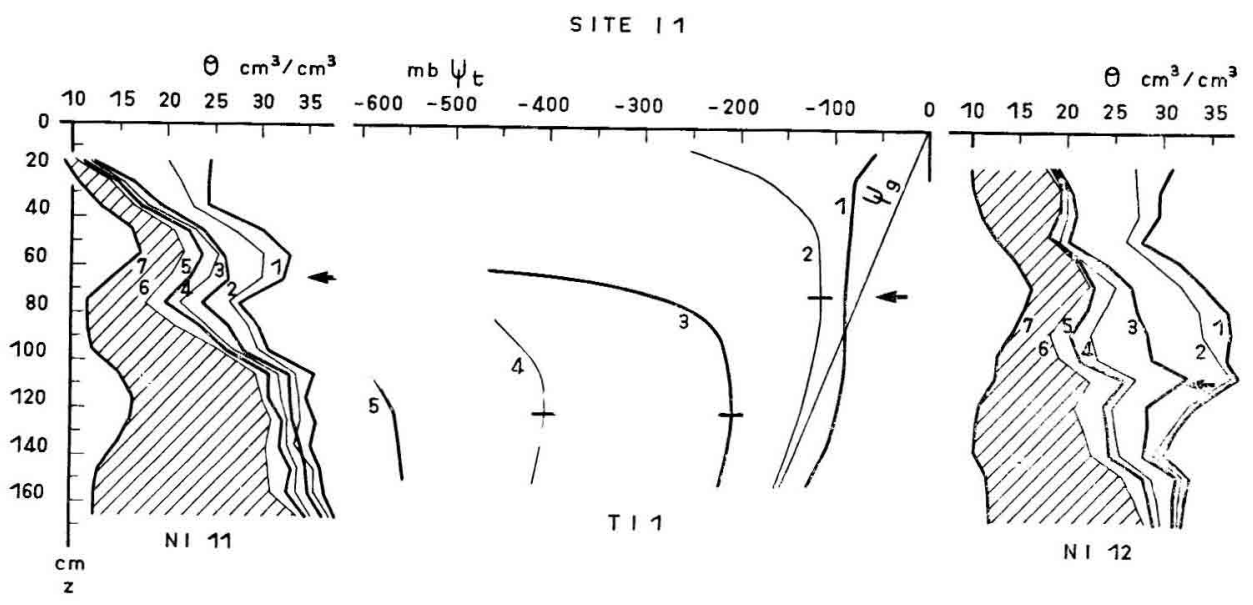

SITE I 2
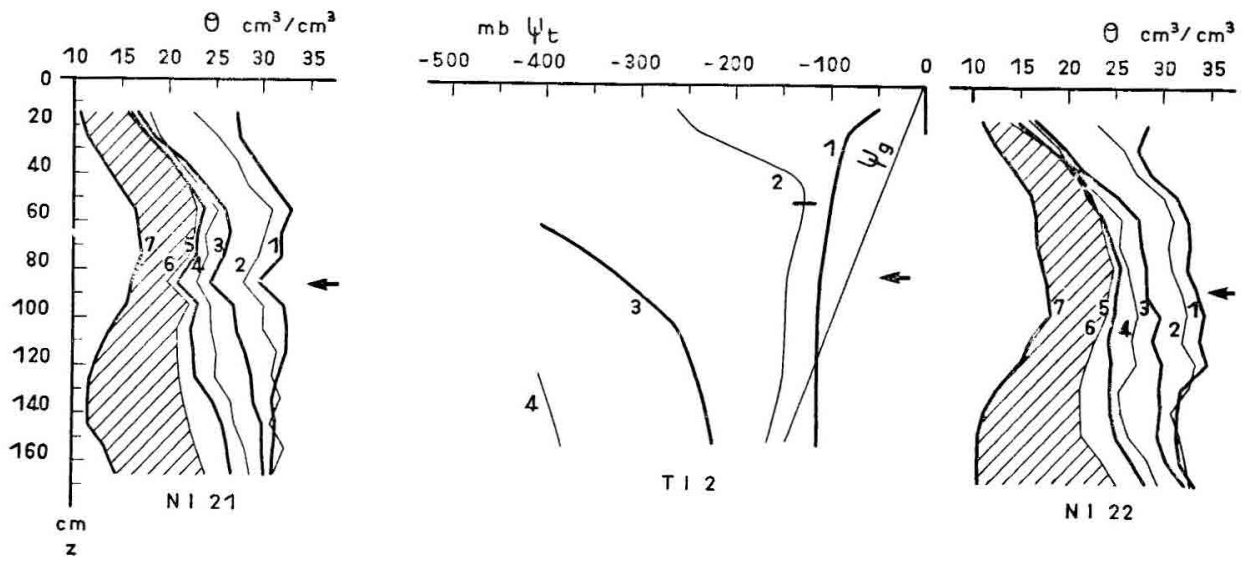

N I 22

Frg. $13 \mathrm{~A}$ 
SITE I 4
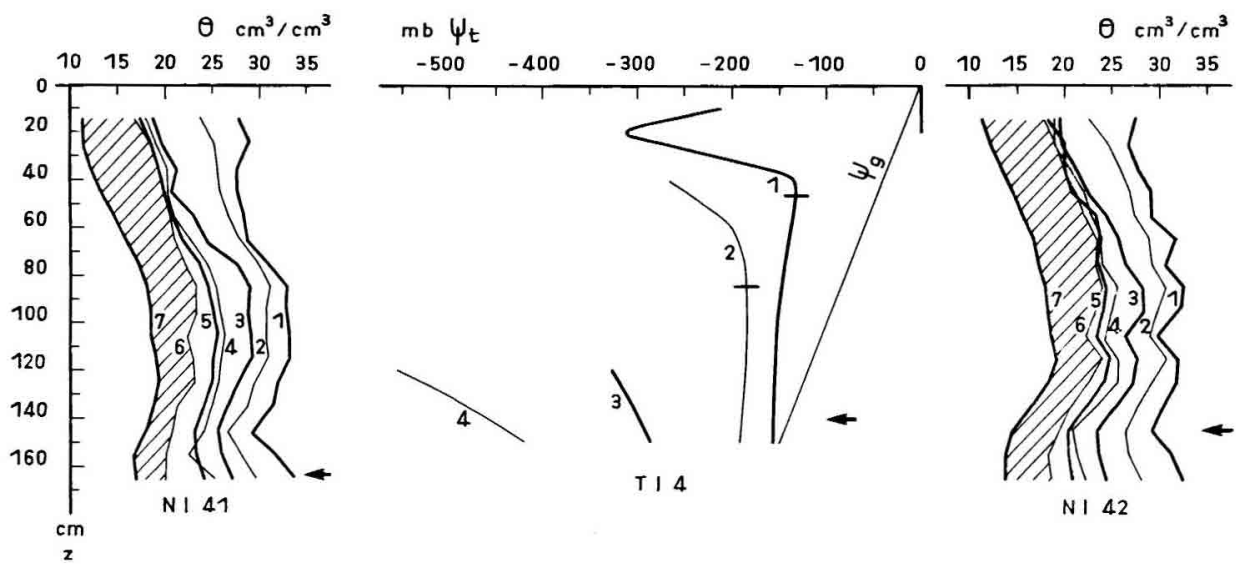

NI 42

SITE I 5
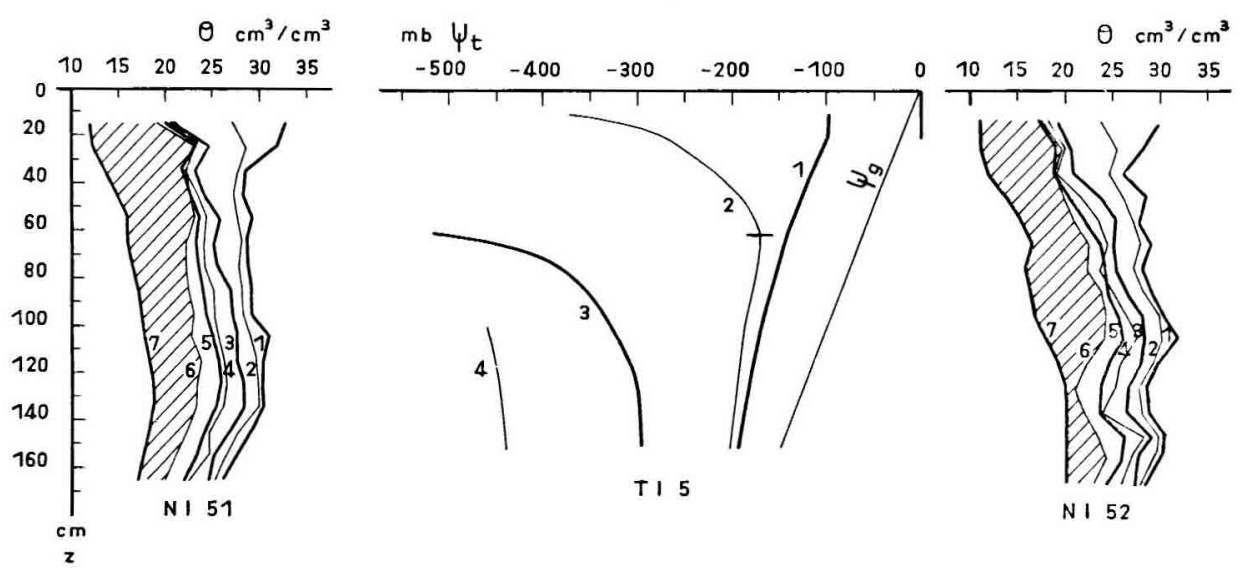

FIG. 13 B

Fig. $13 \mathrm{~A}$ et $\mathrm{B}$

Evolution des profils du potentiel hydrique total $\left(\mathbf{W}_{t}\right)$ et d'humidité volumique ( $\left.\theta\right)$ au cours de la petite saison sèche de 1981 et de la grande saison sèche de 1980. Les flèches indiquent le niveau d'apparition du matériau d'altération de la pegmatite. Evolution of the total water potential profiles $\left(\Psi_{t}\right)$ and of soil moisture profiles $(\theta)$ during the short dry-season of 1981 and during the long dry-season of 1980. The arrows indicate the appearance of the altered rock material.

1 : 6 mars 1981 .

$2: 15$ mars 1981 .

3 : 30 mars 1981 .

$4: 23$ septembre 1980 .
$5: 30$ septembre 1980.

$6: 5$ novembre 1980 .

7 : $\mathrm{pF} 4,2$.

Concernant les mesures neutroniques, on notera la très bonne correspondance entre les deux tubes d'accès d'un même site tant pour la forme des profils verticaux (relation avec l'organisation pédologique) que pour leur évolution au cours du temps, 


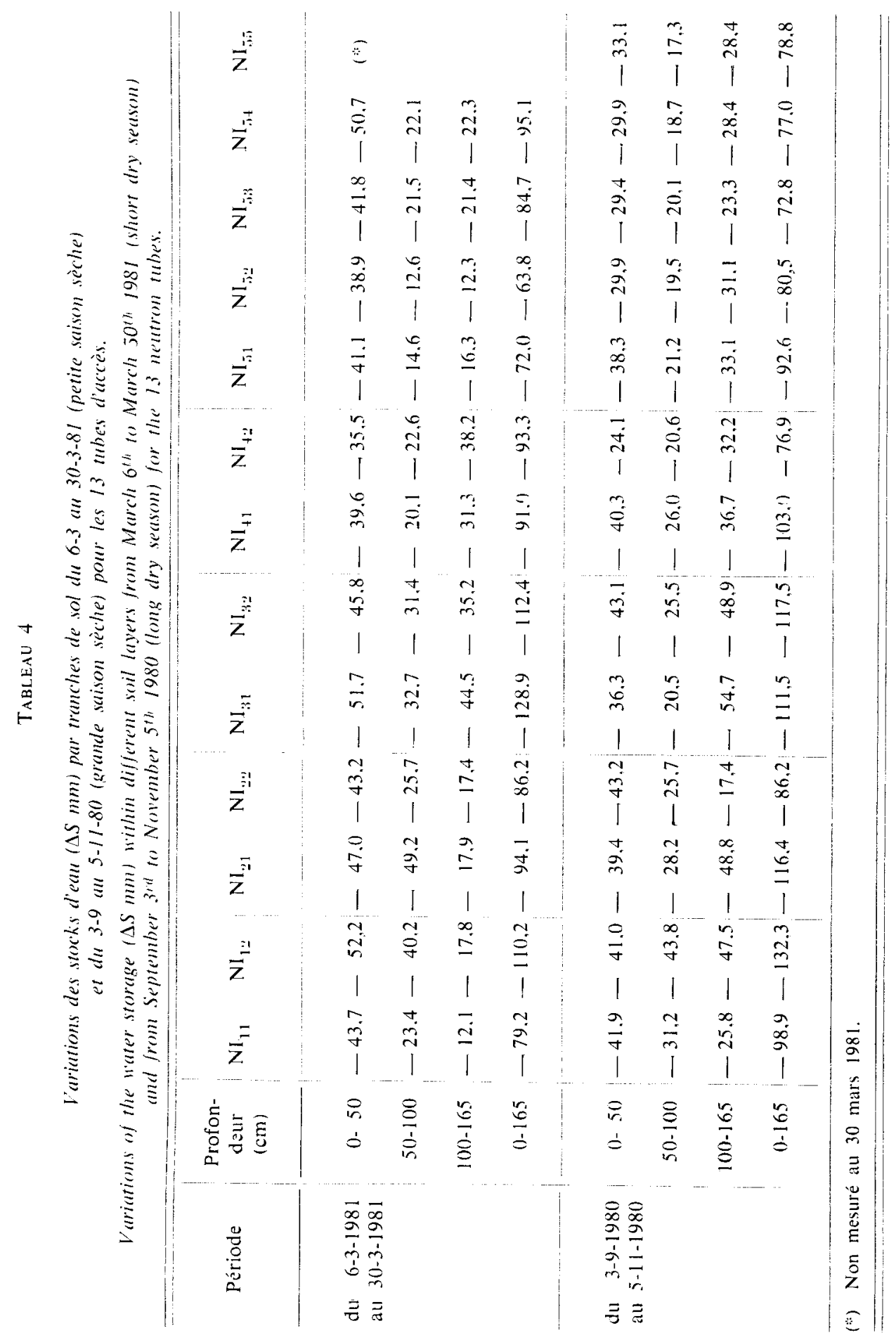


exception faite toutefois du site $I_{1}$ où le lube $N I_{1.2}$ correspond à une invagination du matériau des horizons supéricurs dans le matériau d'altération, les variations d'humidité dans le temps y sont particulièrement imporlantes (échanges latéraux?). On retiendra done comme seul représentatif du site $I_{1}$ le site $\mathrm{NI}_{11}$.

Le tableau 4 présente pour les 15 tubes daccès les variations de stock d'eau pour les tranches de sol 0-50, 50-100 et 100-165 cm du 6-3-1981 au 30-3-1981 (petite saison sèche) et du 3-9-1980 at 5-11-1980 (grande saison sèche).

\subsection{Au cours de la petite saison sèche de mars 1981}

Deux types bien tranchés d'évolution des profils d'humidité apparaissent sur la figure 13 (profils 1,2 et 3 ).

- $I_{1}, I_{2}$ et $I_{5}$ : les variations d'humidité affectent l'ensemble de la partie prospectée du profil mais décroissent avec la profondeur. Cette décroissance est très nettè dès $80 \mathrm{~cm}$ en $I_{11}$, n'est marquée en $I_{.2}$ qu’à partir de $120 \mathrm{~cm}$ et est très progressive en $I_{i}$.

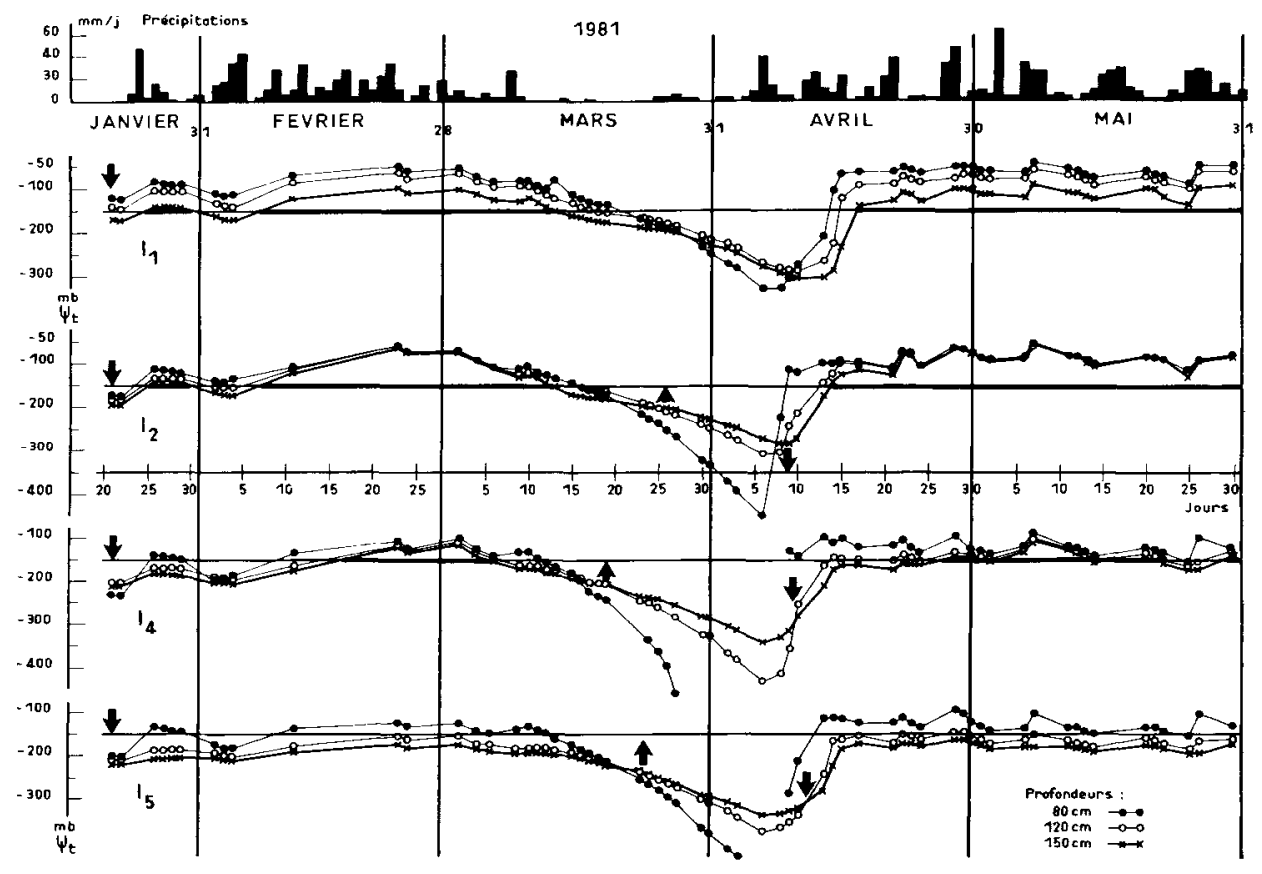

FI(i. 14

Exolution du potentiel hydrique total aux cotes : $80 \mathrm{~cm}(\bullet-\bullet), 120 \mathrm{~cm}(0-0)$

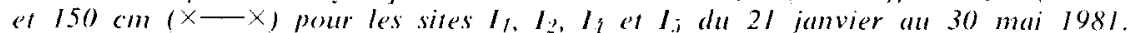
Les fleches verticales indiquent le sens des flux verticaux entre 120 et $150 \mathrm{~cm}$.

Evolution of the total water potential at $80 \mathrm{~cm}(-\longrightarrow), 120 \mathrm{~cm}(\mathrm{O}-\mathrm{O}$

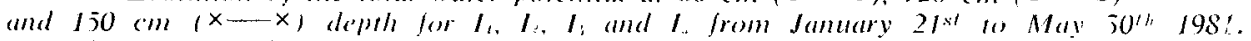

The arrous indicate the direction of the vertical fluxes between 120 and $150 \mathrm{~cm}$. 
- $I_{1}$ : le décalage en profondeur des profils est très important et s'observe (passage de 1 à 2) alors même que la partie profonde du profil reste en régime de drainage (profils de $\Psi_{t}$ ). Cela traduit très probablement un flux de drainage vertical important lié à la présence des horizons 4 et 5 sableux à forte macroporosité (fig. 7) et de $K_{*}$ élevé (fig. 5).

Lal représentation du champ du potentiel hydrique total au 24 mars 1981 (fig. 15) montre une opposition nette entre d'une part les sites amont $I_{5}, I_{1}$ et $I_{3}$ fonctionnant «en remontées capillaires» sur l'ensemble prospecté du profil (représentation des Équipotenticlles non permise en raison des discontinuités inter-sites) et d'autre part

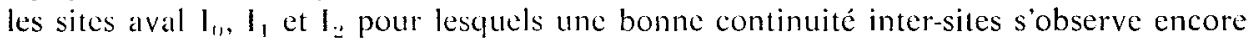
avec existence d'une surface de "flux nul» à lintérieur du matériau d'altération.

L'analyse des données présentées par la ligure 14 confirme cette opposition.

Sur cette figure, nous avons représenté l'évolution de $\Psi$, de janvier à fin mai 1981 en $I_{1}, I_{2}, I_{4}$ et $I_{-}$el aux profondeurs 80,120 et $150 \mathrm{~cm}$. Cette représentation permet une lecture rapide du sens et de l'importance des gradients verticaux de $\Psi^{\prime}$, (nous avons représenté par des flèches verticales le sens des flux entre les cotes 120 et $150 \mathrm{~cm}$ ) el de la présene ou de l'absence de la nappe au-dessus de la cote $150 \mathrm{~cm}$ (rappel par un trait horizontal épais de la valeur de $\Psi_{\infty}$ à cette profondeur lorsque $\left.\Psi_{n}=\Psi_{1}-\Psi_{w}>0\right)$.

Mais, intéressons-nous ici à la cinétiq̣ue de dessèchement-réhumectation liée au "petit élé de mars"s.

En $I_{1}$ (et aussi en $I_{4}$ ), le non croisement des courbes relatives aux cotes 120 et $150 \mathrm{~cm}$ indique un fonctionnement restant qualitativement en drainage pour lensemble du petit été de mars.

En $1 .$. , on note entre 120 et $150 \mathrm{~cm}$ le passage à un régime de remontées capillaires à compler du 27 mars. Lal réhumectation (réaugmentation de $\Psi_{t}$ ) est amorcée à $120 \mathrm{~cm}$. après recroisement des courbes 80 et $120 \mathrm{~cm}$ et à $150 \mathrm{~cm}$ après recroisement des courbes 120 et $150 \mathrm{~cm}$ et est done à relier ici à la réapparition du drainage. Ce dernier comportement s'observe également en $I_{1}$ (fig. 14) el en $I_{1}$ (non représenté) pour les niveaux 80 et $120 \mathrm{~cm}$.

En $I_{1}$ et $I_{5}$. le passáge au régime de remontées capillaires se fait respectivement dès le 19 et le 24 mars. Mais, à la différence du site $I_{2 .}$. on note dans ces deux cas une réaugnentation de $\Psi_{1}$ à $150 \mathrm{~cm}$ de profondeur avant même le rétablissement du drainage. Cette réaugmentation est donc à attribuer au flux de remontées capillaires. Ce cas de figure s'applique Également at site $I_{::}$.

\subsection{Aa cours de la grande saison sèche de 1980}

Par rapport aux profils 3 (situation la plus « sèche» du petit élé de mars) des figures $13 \mathrm{~A}$ et $\mathrm{B}$, les profils 4,5 et 6 relatifs à la grande saison sèche de 1980 montrent des variations d'humidité alfectant essenticllement les profondeurs supérieures à $50 \mathrm{~cm}$.

Au-dessus de cette prolondeur on note unc diminution très importante des variations d'humidité traduisant un freinage des possibilités d'extraction racinaire, bien 
que l'humidité du sol soit encore nettement supérieure (exception faite du site $I_{11}$ ) à celle caractérisant le $\mathrm{pF} 4,2$.

Les variations importantes d'humiditć affectant jusqu'à la profondeur $165 \mathrm{~cm}$ les sites $I_{5}, I_{1}$ et $I_{2}$ indiquent une exploitation des réserves hydriques dépassant largement cette profondeur.

En $1_{1}$, comparativement à ces dermiers sites, les variations d'humidité entre les profils 3 et 6 sont nettement plus faibles au-delà de $90 \mathrm{~cm}$ de profondeur pour $I_{11}$ et

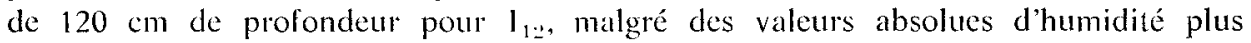
élevées. Le plan de flux nul ne dépasse ici la cote $150 \mathrm{~cm}$ qu'à partir du 30 septembre.

\section{Discussion}

\section{En sillation d'exces d'eatu}

Le fonctionnement hydrodynamique du système étudié confirme largement les résultats de l'étude pédologique (Boul.rT, 1981). La partie amont du transect ( $I_{5}$ ) correspondant à la couverture ferrallitique intiale se caractérise par un icoulement vertical non saturé (fig. 8 et 10), même au plus fort des saisons des pluies. Vers l'aval, le fort contraste de perméabilité lié à la remontée vers la surface de l'horizon 6 (matériau d'altération de la pegmatite) provoque lapparition d'un écoulcnent latéral en milieu saturé (nappe perchée).

Les essais effectuées à l'appareil de Muntz font apparaître une absence totale d'écoulement vertical à travers l'horizon 6 (fig. 5), mais la perturbation du sol par l'enfoncement du double cylindre (destruction de la structure du sol) et le possible colmatage des pores lié à la surcharge d'eau ont pu empêcher la mise en évidence des valeurs non tout à fait nulles de $K_{*}$.

Dans de telles conditions d'écoulement vertical très faible ou inexistant, l'écoulement de la nappe est lić à la configuration topographique interne du matériau plancher (fig. 10). Dans le système étudié, cet écoulement latéral est important (fig. 12). Cependant, en cas de discordance entre matériau plancher et la topographie de surface, il y a possibilité d'écoulement à contre-pente (un tel écoulement est très probable entre $I_{1}$ et $I_{5}$ (fig. 3) ou de piégeage deau dans des cuvettes du matériau plancher pouvant d'ailleurs constituer localement un intéressant report d'eau sur les périodes de déficit hydrique.

Au plan méthodologique, l'utilisation des tensiomètres apparaît comme un intéressant outil d'investigation poul l'étude de systèmes hydropédologiques de pente plus puissant que l'utilisation de tubes piézométriques (LıVET, 1976). puisque permettant également l'étude de la zone non saturée et des relations entre zone saturée et non saturée (fig. 9, 10 ct 11 ). Le niveau dintégration spatiale permis par la représentation bidimensionnelle du champ du potentiel hydrique total (fig. 9, 10 et 11) constitue ainsi un maillon intermédiaire intéressant entre les études hydrologiques à l'échelle du bassin versant et les observations pouvant être faites au niveau de chaque site. 


\section{Evolution des profils hydriques en saisons sèches et système racinaire}

Au cours des deux saisons sèches, caractérisées par d’importants déficits hydriques climatiques (tabl. 3), le réservoir sol est très largement utilisé pour la satisfaction des besoins hydriques de la forêt (tabl. 4 et fig. 13). Les variations d'humidité affectent une épaisseur importante de sol, cela confirme les observations similaires faites en forêt primaire par DUCRl:y (1981) non loin de notre transect d'étude (sols sur schistes) et par Huttel. (1975) ainsi que par BoIs \& Rosf (1978) en milieu ferrallitique de Basse Côte-d'lvoire. L'utilisation de tensiomètres, indiquant le sens des llux d'eau dans le sol, permet daffirmer ici que ces variations profondes sont pour leur grande part à attribuer au llux évapotranspiratoire, le drainage vertical n'intervenant significativement quen début des saisons sèches et uniquement dans les zones à DVL (cf. 3.231.).

Hormis le cas du site $I_{1}$ que nous aborderons plus loin, les deux profils extrêmes 1 et 6 présentés par la figure 13 sont sensiblement parallèles pour la profondeur de sol prospectée par les tubes d'accès. Il n'en est pas de même des profils intermédiaires $(2,3.4 \mathrm{et}$ ) présentant un décalage affectant progressivement des profondeurs de plus en plus importantes.

Une telle image d'évolution des profils d'humidité, s'opposant à une évolution par simple dessèchement des couches superficielles sans variations en profondeur telle celle observée par Aussinac \& Graniar (1979) dans une futaie de l'Est de la France caractérisée par un enracinement superficiel, el se rapprochant de limage de translalion continue des profils observée palr MIRIAUX \& BERTRAND (1976) en conditions d'exploitation racinaire profonde par la vigne dans le midi de la France, atteste d'une extraction racinaire dépassant largement la profondeur des tubes d'acees $(1.65 \mathrm{~m})$.

Des observations analogues aux nôtres. d'un décalage progressif vers le bas d'un front d'extraction racinaire maximale ont été faites par Mahboubi (1980) sur graminées et peuvent être interprétées a la fois par une augmentation de la résistance au transfert de l'eau entre le sol et les racines et par une diminution de la différence de potentiel hydrique entre le sol et les racines au cours du dessèchement intervenant bien avant le $\mathrm{pF}^{4}$ 4.2, le maximum d'extraction racinaire se décalant alors en profondeur vers une zone plus humide présentant une résistance au transfert de l'eau et une différence de potentiel sol-racine plus favorable.

Ces observations d'extraction racinaire profonde peuvent surprendre dans le cas d'une forêt tropicale humide à laquelle on attribue généralement un fonctionnement racinaire superficiel.

Toutefois les profils d'enracinement établis en Guyane par Humbel (1978) montrent qu'en zone de drainage vertical libre on trouve, malgré une forte concentration superficielle, encore près de 5 p. 100 (en poids de matière sèche) de racines, surtout tines, uniformëment réparties entre 1 et $2 \mathrm{~m}$ de profondeur (mesures non poursuivies au-delà de $2 \mathrm{~m}$ ). Dans la zone étudiće, nous avons pu observer de notre côté la présence de racines bien au-delà de $2 \mathrm{~m}$ de profondeur. Nos résultats montrent donc que ces racines profondes, en dépit de leur faible densité, jouent un rôle important dans l'alimentation hydrique des arbres tout au moins durant la grande saison sèche.

Il importe par ailleurs de remarquer que les mesures massiques de densité raci- 
naire privilégient beaucoup trop les horizons superficiels où se trouvent de grosses racines. Sur le plan hydrique mieux vaudrait s'intéresser aux longueurs de racines par unité de volume de sol (MahBoubi, 1980).

En $I_{1}$ les variations d'humidité en profondeur sont nettement plus faibles que pour les autres sites, cela est à relier en $\mathrm{I}_{11}$ à la présence dès $80 \mathrm{~cm}$ de profondeur $\left(110 \mathrm{~cm}\right.$ en $\left.I_{1.2}\right)$ du matériau d'altération réduisant les possibilités de développement du système racinaire (Humbel, 1978). Toutefois la diminution continue dans le temps de l'humidité (fig. $13 \mathrm{~A}$ ) observée jusqu'a $165 \mathrm{~cm}$ de profondeur attesic encore de la présence de racines.

Les variations d'humidité très importantes notées en $1_{2.2}$ à l'intérieur du matériau d'altération peuvent, selon toute vraisemblance, être attribuées au réseau de faces lissées caractérisant le sommet de l'horizon 6 (fig. 3) permettant une colonisation racinaire du matériau (observations faites sur le terrain).

\section{Estimation de lévapotranspiration réelle de la lorêt}

La longueur trop faible des tubes daccès ne permet pas ici une telle estimation (sauf probablement une approximation légèrement par défaut en $I_{1}$ ) nécessitant la prise en considération d'un volume de sol intéressant une épaisseur $z$ de sol supéricure au domaine de prospection racinaire. Il nous semble toutefois utile de préciser les conditions d'une telle estimation dans le milieu étudié.

La condition ci-dessus énoncée étant supposée remplie, le bitan hydrique s'écrit pour une période considérée:

$$
-\Lambda \mathrm{S}=\mathrm{ETR}-\mathrm{Pi}+\mathrm{D}+\mathrm{R}
$$

avec IS: variation du stock d'éau dans le volume considéré

ETR : évapotranspiration réclle du couvert forestier y compris linterception par le couvert

$\mathrm{Pi} \quad$ : précipitations incidentes au-dessus du couvert

D : bilan des flux hydriques souterains (flux latéraux et flux verticaux à la profondeur z)

$\mathrm{R} \quad$ : bilan de ruissellement

Seuls les termes $\Delta S$ et $P i$ sont facilement accessibles en général. En ce qui concerne le ruissellement on devra s'assurer que $\mathrm{R} \simeq 0$ (faible pente, périodes de faibles précipitations, sols à drainage vertical libre), sinon $R$ devra être évalué.

En ce qui concerne les écoulcments souterrains latéraux, on vérifiera que pour notre transect d'étude alors même que la nappe n'a pas totalement disparu en profondeur (fig. 11), les flux deviennent verticaux descendant dans les horizons supérieurs puis s'inversent rapidement avec l'entrée dans la saison sèche pour devenir remontants et parallèles à la surface du sol (fig. 15). Les gradients latéraux de $\Psi_{t}$ sont alors faibles (compte tenu de la non correspondance des échelles horizontales et verticales) (cf. 3.222.) et affectent essentiellement l'horizon 6 de très faible conductivité hydraulique. 


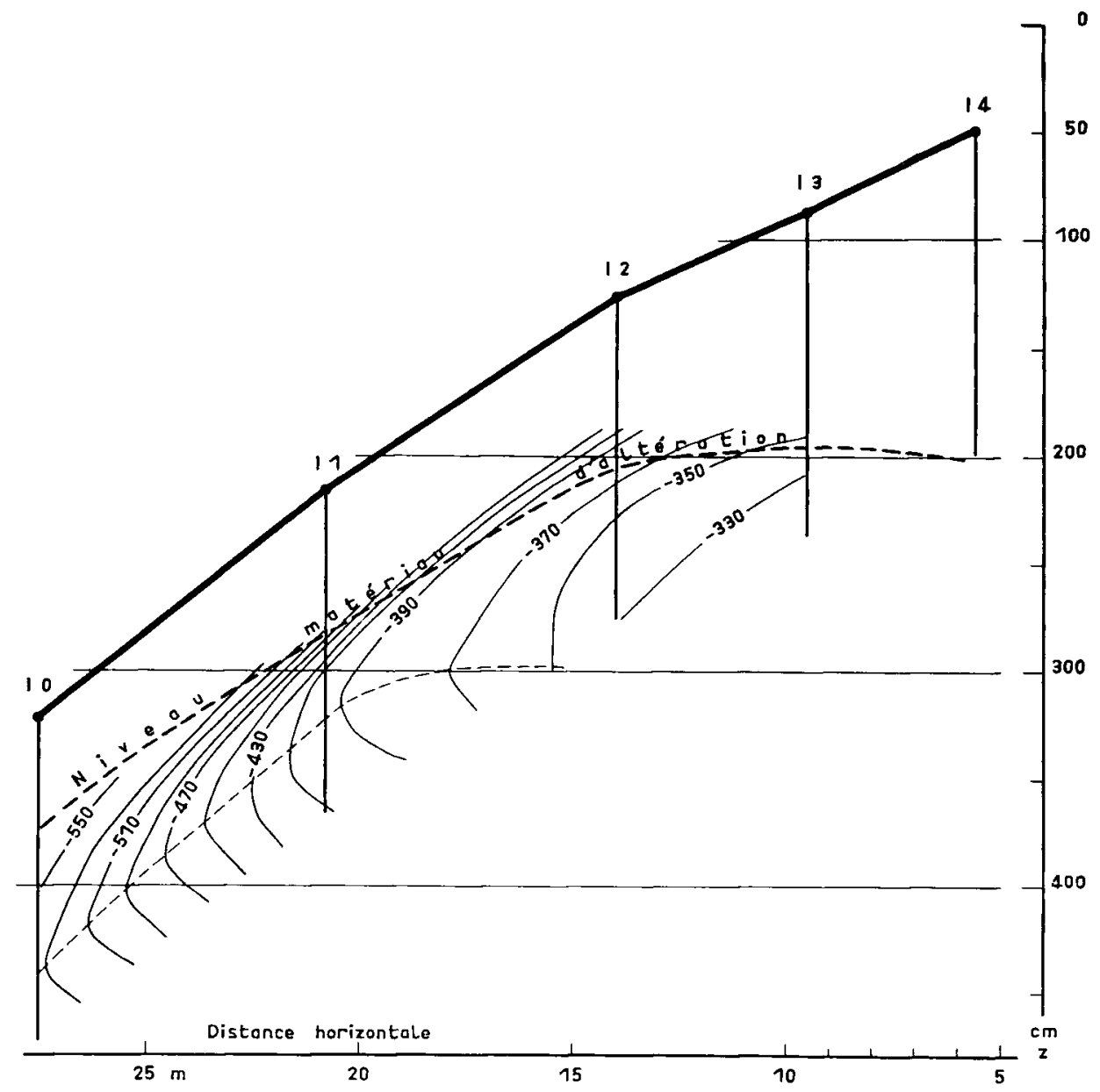

FIG. 15

Champ du potentiel hydrique total $\left(\Psi_{f}\right)$ au cours de la petite saison sèche (24 mars 1981). Mêmes conventions que figure 9. (- - ) : surface de flux vertical nul.

Field of the total water potential $\left(\Psi_{1}\right)$ during the short dry-season (March 24th 1981 ). Same conventions as in figure 9. (-- -) : surface of vertical zero flux.

L'équation (13) devient alors, si l'on suppose $\mathrm{R}=0$ :

$$
-\Delta \mathrm{S} \simeq \mathrm{ETR}+\mathrm{D}_{\mathrm{v}}
$$

où $D_{v}$ est le bilan des flux verticaux (drainage ou remontées capillaires) à travers la cote de contrôle $z$.

- Pour les zones à drainage vertical bloqué on pourra supposer $\mathrm{D}_{\mathrm{v}} \simeq 0$, donc

$$
-\Delta \mathrm{S} \simeq \mathrm{ETR}
$$


- Pour les zones à drainage vertical libre, il sera nécessaire de déterminer $D_{v}$ par application de la loi de Darcy [équation (6)] à la cote z. Cette détermination

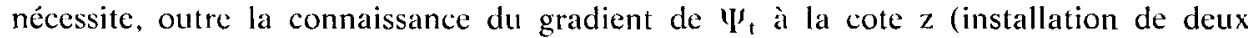
tensiomètres profonds de part et dautre de cette cote), la connaissance de la relation $\mathrm{K}_{\%}(0)$ en conditions non saturées.

En dehors du recours aux méthodes de détermination de $\mathrm{K}$ en laboratoire sur échantillons de sol (Hultel, 1974) nécessitant un appareillage spécial et souvent biaisées par rapport aux valeurs in situ (JAILLARD, 1980), des solutions de terrain peuvent être envisagées, basées sur des mestures neutroniques et tensiométriques, ces méthodes supposent l'absence d'extraction racinaire.

- La méthode du plan de flux nul (Daudet \& Vachaud, 1977) apparaît, en dehors des situations dans lesquelles est prévue une coupe rase, comme impossible à mettre en œuvre en forêt, l'évaporation au niveau du sol étant trop faible dans les conditions microclimatiques du sous-bois.

- La méthode de drainage interne (HıLlec et al.. 1972) peut être envisagée en forêt pour les sols bien draînants, mais à condition d'effectuer les essais dans un monolithe (lBRAHIM, 1979; VACHAUD et al.. 1981) afin de supprimer l'extraction racinaire. La méthode devenant alors destructrice, il faudra travailler soit en un site voisin de celui des mesures (risques d'erreurs liés à l'hétérogénéité spatiale des caractéristiques du sol), soit en ce même site après les campagnes de mesures.

Pour la caractérisation des horizons profonds, il pourra être intéressant pour obtenir des variations d'humidité importantes, d'effectuer l'essai non pas à la surface du sol mais au fond d'une fosse.

\section{Conclusion}

Au plan de la démarche utilisée on soulignera l'intérêt qu'il y a à déterminer les sites de mesure en fonction de l'organisation morphologique de la couverture pédologique étudiée et préalablement connue de façon détaillée. Ceci permet d’une part de référer les mesures à cette organisation, d'établir la relation entre ce que l'on pourrait appeler «l'anatomie» et la «physiologie» de la couverture pédologique, de faciliter la compréhension des mécanismes mis en auvre, et d'autre part d'extrapoler les comportements ainsi précisés à l'ensemble des sites morphologiquement identiques.

En situation d'excès d'eau, le fonctionnement hydrodynamique du système étudié s'accorde bien avec les résultats de l'étude des caractéristiques du matériau et constitue une confirmation expérimentale de la distinction entre sols à drainage vertical libre et sols à drainage vertical bloqué.

Concernant la détermination précise des termes du bilan hydrique en saison sèche, cette étude aura surtout été l'occasion d'une mise au point méthodologique adaptée au milieu forestier guyanais. Toutefois, certains traits originaux du fonctionnement hydrique du système étudié ont pu être dégagés. Ainsi, lors des périodes de 
déficit hydrique, l'exploitation des réserves hydriques du sol est profonde et semble liée à des possibilités d'extraction racinaire profonde.

Limportance des dilférences observées dans le fonctionnement hydrique in situ des deux types de sol étudiés et des répercussions en termes de conditions de la production végétale liées à ces différences (conditions pédoclimatiques, disponibilités en eau, entraînement des éléments chimiques et notamment des fertilisants...) justifierait pleinement la poursuite des recherches entreprises dans le but d'une détermination précise des termes du bilan hydrique. Ces recherches devraient être menées à la fois en situation de forêt primaire et pour les différents scénarios de mise en valeur du milieu après déforestation (étude des interactions type de sol - type d’aménagement).

\title{
Remerciements
}

Cette étude a été réalisée grîce au support financier de la D.G.R.S.T. (Décision d’aide n" 79-7-(1)435 et 79-7-(0436, Comité Scientifique Gestion des Ressources Naturelles Renouvelables).

Je tiens à remercier MM. Boultet, Fritsch \& Lucas (O.R.S.T.O.M. Guyane), MM. Du(RI:Y \& VALANCOGNi: (I.N.R.A. Antilles-Guyane) et M. SARRAILH (C.T.F.T. Coordomnateur de lopération ECEREX) pour l'aide précieuse quils mont fournie tout al long de ce travail. ainsi que M. Henrion (C.N.R.F. Champenoux) pour la délicate réalisation des figures.

\author{
Summary \\ Soil water dynamic in the tropical rain forest of French Guyane. \\ Influence of the soil cover
}

In French Northern Guyane the detailed study of the soil cover has focused attention on the existence of soil systems with large lateral differenciation which have been interpreted as being transformation systems of an initial ferralitic cover resulting from a slight tectonic rise of the land (a few meters) (Bouler et al.. 1979).

In the present study (part of the ECEREX pluridisciplinary study; Sarranth, 1980) our purpose was to evaluate the repercussions of that environmental variability on the terms of the soil hydrologic cycle. The experimentations (iit-situ neutronic and tensiometric measurements) concerned a mixed soil system (pegmatitic rock-material) in a slight sloping situation.

During the rain seasons, in the initial ferralitic cover $\left(I_{i}\right.$, fig. 3 ), the existence of hight macroporosity values (fig. 7) for the microaggregated horizons 1 and 2 (fig. 3) and of a permability coefficient (Muntz method) which remained superior to $3 \mathrm{~mm}^{1^{-1}}$ in the two upper meters of soil (fig. 5) allowed an unsaturated vertical drainage even during periods of important rainfall (fig. 8, 10).

From $I_{4}$ to $I_{6}$, the rise towards the soil surface of horizon 6 (incision of the initial topographic surface) characterized by high bulk density (tabl. 1) and small macroporosity values (fig. 7) and by near zero permeability coefficient values (fig. 5) led, during the rain seasons, to the appearance of a perched water table (fig. 8) with an efficient lateral drainage component (fig. 10,12) due to a favourable internal configuration of the top of horizon 6 (fig. 3). 
During the two studied dry-seasons (short dry season in March 1981 and long dry. season from August to November 1980) characterized by important climatic water deficits (tabl. 3), the soil water reserve was widely used for the forest evapotranspiration (tabl. \& and lig. 13). Two different patterns of deep soil-water content evolution appeared :

- From $I_{5}$ to $I_{2}$ (fig. 3) the important water content variations which could be observed down to a $165 \mathrm{~cm}$ depth, indicated that the water uptake by roots was deeper than the neutron tubes.

- For the $I_{1}$ site, the deep water content variations were notably smaller, which could be attributed to the presence of horizon 6 in which the root sy'stem development is hindered.

In such conditions of deep water-uptake by roots it was not possible here to make an estimate of the forest evapotranspiration with the neutronic measurements, but the experimental conditions which should allow such an estimate are specified.

Reçle le 30 ochobre 1982.

Acceple le 29 arril 1983.

\section{Références bibliographiques}

Aussenac G., Granier A., 1979. Etude bioclimatique d'une futaie feuillue (Fagas silvarica L. et Quercus sessiliflora Salisb.) de l'Est de la France. ll. Etude de l'hunidité du sol et de l'évaporation réelle. Ann. Sci. For, 36 (4), 265-280.

Bal.t. J.L., 1980. Caractères et réserves hydriques des sols sur craie et graveluche en Champagne. Ann. Agron., 31 (4), 473-485.

Bors J.F., Roost: E.J., 1978. Réflexions sur les résultats de mosures systématiques d'humiditć à la sonde à neutrons dans un sol ferrallitique de Basse Côte-d'Ivoire. Cah. O.R.S.T.O.M.. Sér. Hydrol., 15 (4), 351-364.

Bour.ET R., 1978. Existence de systèmes à forte différenciation latérale en milien ferrallitique guyanais : un nouvel exemple de couvertures pédologiques en déséquilibre. Sci. Sol., Bull. A.F.E.S., 2, 75-82.

Bouter R., 1981. Etude pédologique des bassins versants ECEREX. Bilan de la cartographic. Bull. Liaison ECEREX, n" 4, O.R.S.T.O.M. Cayenne, 4-22.

Boulet R., Brugiere J.M., Humbel. F.X., 1979. Relations entre organisation des sols et dynamique de l'eau en Guyane française septentrionale : conséquences agronomiques d’une évolution déterminée par un déséquilibre d’origine principalement tectonique. Sci. Sol. Bull. A.F.E.S., 1, 3-18.

Brochet P., Glikbuer N., 1975. L'évapotranspiration. Aspect agrométéorologique. Evaluation pratique de l'évapotranspiration potentielle. Monogr. Météorol. Natl., n“ 65, $95 \mathrm{p}$.

Brutsaket W., 1966. Probability laws for pore-size distributions. Soil Sci., 101 (2), 85-92.

CosANIJE C.M., 1978. Etalonnage d'un humidimètre a neutrons dans un sol forestier. Bulletin du G.F.H.N., $\mathrm{n}^{0}$ 3, 25-35.

Couchat P., 1977. Aspects méthodologiques et technologiques de la mesure neutronique de l'humidité des sols. Ann. Agron., 28 (5), 477-488.

Daudet F.A., Vachaud G., 1977. La mesure neutronique du stoch d'eau du sol et de ses variations. Application à la détermination du bilan hydrique. Ann. Agron., 28 (5), 503519.

Ducrey M., 1981. Etude de l'humidité et de la réscrve en eau des sols des bassins versants F, G et H. Bull. liaison ECEREX, n" 4, O.R.S.T.O.M. Cayenne, 61-65.

Fougierouze J., 1965. Le climat de la Guyane française. Type de temps - saisons et régions climatiques. Monogr. Météorol. Natl., n"38,36 p. 
Fougerouze J., 1966. Quelques problèmes de bioclimatologie en Guyane française. Agron. Trop, 3, 291-345.

Fritsch J.M., 1981. Ecoulement et érosion sur les bassins versants ECEREX en 1979. Bull. liaison ECEREX, n" 4, O.R.S.T.O.M. Cayenne, 23-44.

Hillel D., 1974. L'eau et le sol. Principes et processus physiques. Vander éditeur, $288 \mathrm{p}$.

Hillel D., Krentos D., Stylanou Y., 1972. Procedure and test of an internal drainage method for measuring soil hydraulic characteristics in situ. Soil Sci., 114 (5), 395-400.

Humbel F.X., 1978. Caractérisation par des méthodes physiques, hydriques et d'enracinement, de sols de Guyane française à dynamique de leau superficielfe. Sci. Sol. Bull. A.F.E.S., 2, 83-93.

Huthli C., 1975. Recherches sur l'écosystème de la forêt subéquatoriale de Basse Côted'Ivoire. IV. Estimation du bilan hydrique. La Terre et la Vie, 29, 192-202.

IBRAHIM M.M., 1979. Recherches sur la dynamique et le bilan de leau d'un écosystème à Pin pignon (Pinus pinea L.) sur sable dunaire. Thèse d'Etat, Université des Sciences et Techniques du Languedoc, $256 \mathrm{p}$. + annexes.

Jalleard B., 1980. Fonctionnement hydrique d'un sol carbonate. Incidence sur la chlorose ferrique. Thèse de 3" eycle. Université des Sciences et Techniques du Languedoc, 135 p.

Lescure J.P., 1981. La végétation et la flore dans la région de la piste de St-Elie. Bull. Liaison ECEREX, n" 3, O.R.S.T.O.M. Cilyenne, 4-24.

L,IVI:T M., 1976. Etude hydrogéologique des formations de pente de la butte d'Amance. Rapport de recherche n" 52, Ministere de l'Equipement, 106 p.

MADEC H., 1963. L'évapotranspiration potenticlle et le bilan de l'eau en Ciuyane, Monogr. Métésrol. Natl., n" 39, 17 p.

Manbouns A.A., 1980). Etude in situ du bilan hydrique pour quelques graminées : extraction de l'eau par lé sjstème racinaire et résistance à la sécheresse. Thèse DocteurIngénieur, Université des Sciences et Techniques du Languedoc, $174 \mathrm{p}$. + annexes.

Prevost M.F., Puig H., 1981. Accroissement diamétral des arbres en Guyane : observations sur quelques arbres de forêt primaire et de forêt secondaire. Bull. Mus. Natl. Hist. Nat., Paris, 4" sér., 3, 1981, Section B, Adansonia, n" 2, 147-171.

Pulg H., 1979. Production de litière en forêt guyanaise. Résultats préliminaires. Bull. Soc. d'Hist. Nat. Toulouse, T. 115, 3-4, 338-346.

Rochr: MA., 1978. Les bassins versants expérinentaux ECEREX en Guyane française. Etude comparative des ćcoulements et de l'érosion sous forêt tropicale humide. Cah. O.R.S.T.O.M., Sér. Hydrol., 15 (4), 365-378.

Sirkall.h J.M., 1980. L'écosystime forestier guyanais. Etude écologique de son évolution sous l'effet des transformations en vue de sa mise en valeur. Bois For. Trop., n" 189, $31-36$.

SARRALlh J.M., 1981. Parcelles élémentaires d'étude du ruissellement et de Yérosion. Analyse des résultats obtenus durant les deux premières campagnes de mesure. Bull. Liaison ECEREX, n" 4, O.R.S.T.O.M. Cayenne, 45-51.

Vachald G., Dancette C., Sonko S., Thony J.L., 1978. Méthodes de caractérisation hydrodynamique in situ d'un sol non saturé. Application à deux lypes de sol du Sénégal en vie de la détermination des termes du bilan hydrique. Ann. Agron., 29 (1), 1-36.

Vachaud G., Vauclin M., Colombani J., 1981. Bilan hydrique dans le Sud tunisien I. Caractérisation expérimentale des transferts dans la zone non saturée. J. Hydrol., 49, $1(2), 31-52$.

Valancogne G., Fouerl A., Aho N., 1977. Contrôle de l'étalonnage basé sur l'étude neutronique d'échantillons de sol. Bull. G.F.H.N., 1, 61-71.

ZASI.AVSKY D., 1964. Theory of unsaturated flow into a non-unilorm soil profile. Soil Sci., 97, $400-410$. 